\title{
Multi-Input Genetic Algorithm for Experimental Optimization of the Reattachment Downstream of a Backward-Facing Step with Surface Plasma Actuator
}

\author{
N. Benard ${ }^{\ddagger 1}$, J. Pons-Prats ${ }^{\S 2}$, J. Periaux ${ }^{\S, * 3}$, G. Bugeda ${ }^{\S, * 4}$, J.P. Bonnet ${ }^{\ddagger 5}$, and E. Moreau ${ }^{\ddagger 6}$ \\ ${ }^{\ddagger}$ Institut PPRIME - UPR 3346 - CNRS - Université de Poitiers - ISAE/ENSMA - SP2MI Téléport2 Bd Marie \& Pierre Curie \\ BP 30179, 86962 Futuroscope Chasseneuil Cedex, France \\ ${ }^{\S}$ International Center for Numerical Methods in Engineering (CIMNE), c/ Esteve Terrades 508860 Castelldefels, Spain. \\ *Universitat Politècnica de Catalunya. Barcelona Tech (UPC), c/ Gran Capità s/n 08034 Barcelona, Spain
}

\begin{abstract}
The practical interest of flow control approaches is no more debated as flow control provides an effective mean for considerably increasing the performances of ground or air transport systems, among many others applications. Here a fundamental configuration is investigated by using non-thermal surface plasma discharge. A dielectric barrier discharge is installed at the step corner of a backward-facing step $\left(\operatorname{Re}_{\mathrm{h}}=30000, \operatorname{Re}_{\theta}=1650\right)$. Wall pressure sensors are used to estimate the reattaching location downstream of the step. The primary objective of this paper is the coupling of a numerical optimizer with an experiment. More specifically, optimization by genetic algorithm is implemented experimentally in order to minimize the reattachment point downstream of the step model. Validation through inverse problem is firstly demonstrated. When coupled with the plasma actuator and the wall pressure sensors, the genetic algorithm finds the optimum forcing conditions with a good convergence rate, the best control design variables being in agreement with the literature that uses other types of control devices than plasma. Indeed, the minimum reattaching position is achieved by forcing the flow at the shear layer mode where a large spreading rate is obtained by increasing the periodicity of the vortex street and by enhancing the vortex pairing phenomena. At the best forcing conditions, the mean flow reattachment is reduced by $20 \%$. This article, with its experiment-based approach, demonstrates the robustness of a single-objective multi-design optimization method, and its feasibility for wind tunnel experiments.
\end{abstract}

\section{Introduction}

$\mathrm{A}$ lthough their limitations and, in most of the cases, in spite of their technical immaturity, flow control devices are still of great interest for the aeronautical industry. The main challenge for industrial companies is the definition of entirely new environment-friendly aircraft. A manner to improve the aerodynamic performances of airfoil with final objective to reduce the emission of greenhouse gases consists of using active technologies to manipulate the turbulent flow developing on the wings and the airframe of the aircraft. The number of studies investigating mechanical, fluidic or plasma actuators is increasing over the years, this confirming the needs for researches focusing on turbulent flow manipulation by active control. In spite of the extensive amount of investigation and work accumulated over the years, flow control is still an active area of research, recently motivated by new technologies such as plasma discharge actuators. Indeed, among a wide list of active systems used as in-lab flow control devices, surface plasma actuators are ones of the potential candidates for improving the performance of commercial or military aircrafts and micro-air vehicles in terms of a reduced drag or an increase of the manoeuvring capabilities. These actuators present a low technical

\footnotetext{
${ }^{1}$ Nicolas Benard, Associate Professor, nicolas.benard@univ-poitiers.fr, AIAA senior member

${ }^{2}$ Jordi Pons-Prats, Associate researcher, jpons@ cimne.upc.edu

${ }^{3}$ Jacques Periaux, Visiting Professor, jperiaux@gmail.com

${ }^{4}$ Gabriel Bugeda, Full Professor, bugeda@cimne.upc.edu

${ }^{5}$ Jean-Paul Bonnet, Research director, jean-paul.bonnet@univ-poitiers.fr, AIAA member

${ }^{6}$ Eric Moreau, Professor, eric.moreau@univ-poitiers.fr
} 
maturity, but they have been identified for their potential for major advances in weight reduction, fast switching capability, and robust solid-state design with no moving parts that can be easily installed on airfoils [1]. Dielectric barrier discharges are the most used non-thermal plasmas for flow control applications. They can be operated by applying an AC high-voltage source to the air-exposed electrode of the actuator [2-5] or by using continuous series of voltage pulses with rising time of about tens of nanoseconds [6-9]. Despites having two different modes of interactions with the natural flow, these two modes can help in reattaching separated flow over inclined airfoil profile [10-16] among other applications. One of the interests of plasma actuators is the principle of electro-mechanical conversion that implies fast energy transfers. By adjusting the electrical parameters of the electrical signal applied to the air-exposed electrode (amplitude, frequency, frequency modulation, duty-cycle), the amplitude, frequency and duty-cycle of the periodic flow fluctuations induced by the plasma can be easily tuned. The accurate adjustment of the forcing conditions is particularly relevant for flow control investigations where optimum forcing conditions have to be identified. Such capabilities as well as the easy implementation of a plasma actuator into an autonomous command system will be exploited in the present work.

This investigation concerns a fundamental flow configuration: the turbulent flow separation downstream of a Backward-Facing Step (BFS). This configuration is well-adapted for defining unsteady flow control approaches because the flow behind a BFS presents a large variety of periodic motions while the location of the separation point is known and stable [17, 18, 19]. A primary periodic organization arrives from the separated shear layer that develops from the separation point. This separated shear layer evolves as a typical mixing layer in its earlier stage of formation with a typical 'shear layer mode' of instability about $\mathrm{St}_{\theta}=0.012$ [17]. This implies the development of vortical flow structures, organized in a periodic manner, that entrain irrotational fluid from the non-turbulent regions bounding the shear layer. Initially formed at high frequency, the vortices can pair reducing the frequency signature of the vortical flow structure with the downstream position [20, 21]. The impact of the coherent flow structures to the bottom wall is then a periodic phenomenon (usually a value about $\mathrm{St}_{\mathrm{Xr}}=0.6$ is reported as in $[18,19,22]$, with $\mathrm{Xr}$ the reattachment location). The reattachment location is highly unsteady partially due to the periodic impact of the vortical flow structures at $\mathrm{St}_{\mathrm{Xr}}=0.6$. However, as it reported in $[22,23,24]$, a flapping motion of the shear layer contributes to the movement of the reattachment point and to the widening of the separated shear layer. The flapping motion is a low frequency phenomenon with frequency signature at about $\mathrm{St}_{\mathrm{h}} \sim 0.02$ [22, 23]. The BFS configuration is thus a rich environment in terms of periodic motions and it offers a lot of flow control scenarios even for actuators producing a low momentum transfer such are surface plasma discharges $[2,3,4,5]$. Plasma actuators have been used in a lot of aerodynamic flow configurations but among them, BFS has only received a recent attention. For instance, the effectiveness of DBD actuator to shorten the recirculation bubble $(-35 \%)$ at low Reynolds number $\left(\mathrm{Re}_{\mathrm{h}}=1520\right)$ and laminar regime has been shown in D'Adamo et al. [25] by forcing the flow with a vertical plasma jet. Studies at a higher Reynolds number (Reh 30000) have been recently published. In [26, 27], a reduction in the recirculation length of about $20 \%$ is observed for homogeneous spanwise unsteady actuation at the step mode of instability $\left(\mathrm{St}_{\mathrm{h}} \sim 0.25\right)$. The unsteady wall pressure signals suggested a strong periodization of the flow structures caused by the imposed periodic fluctuations. Plasma actuator design promoting 3D flow has also been tested on a BFS configuration. In [28], Zare-Behtash et al. confirm that serpentine DBD can add velocity into the initial region of the shear layer, but the influence on the mean reattachment location is not discussed. 
This article presents an original experimental approach for determining the best forcing conditions that minimizes the reattachment location behind a BFS, this in an autonomous and modelfree manner. Indeed, among several applications, optimization by combining evolutionary algorithms and numerical simulations had demonstrated their potential to solve high dimensional problems occurring in fluid dynamics. In a few contributions, evolutionary algorithms are used to optimize the flow control conditions by full numerical approaches. For instance, suction/blowing jet mounted on the upper surface of a NACA0012 airfoil has been numerically optimized in [29]. The optimal forcing mode and more specifically the forcing frequency that maximize the spreading rate of a turbulent jet has been shown in [30] by direct numerical simulation. The problem of time-averaged drag minimization of a circular cylinder by rotary oscillation has been addressed in [31]. Recently, a NSGA-II code has been numerically applied to optimize the flow reattachment along a NACA0015 model by plasma discharge [32]. In the present study, because genetic algorithm only requires the evaluation of the cost function regarding a set of control parameters, such an evolutionary algorithm is used in conjunction with experimental measurements in a feedback loop process. Here, the objective is not to define a function transfer (control law) between the sensors, and thus local or global flow conditions, and the actuator as it has been recently done in $[33,34]$. The present contribution interests in finding the best operating conditions over a large parameter space by coupling for the first time a numerical optimizer and tests conducted in an experimental wind-tunnel. The genetic algorithm is used to solve a single-objective optimization problem (minimization of the reattachment location) with varying voltage amplitude, burst frequency and duty-cycle. In a second part of the paper are introduced time-resolved flow measurements by PIV with the goal to interpret the best forcing conditions determined by the numerical optimizer.

\section{Experimental setup}

The model includes a ramp, followed by a flat plate and ended by a backward step having a height, $\mathrm{h}$, of $30 \mathrm{~mm}$ and a spanwise length of $300 \mathrm{~mm}$. This model is installed in a closed-loop wind-tunnel (Figure 1) having a moderate turbulent intensity (1\%). The dimension of the test section is $300 \times 300 \times 1000 \mathrm{~mm}^{3}$. The model covers the full spanwise of the test section and the expansion ratio equals to 1.1. The aspect ratio (channel width on step height) is larger than 10 this guaranteeing for a two-dimensional flow in the center of the wind tunnel. All measurements are performed for a freestream velocity of $15.6 \mathrm{~m} / \mathrm{s}$. The study is performed for a fully turbulent flow with tripped boundary layer and Reynolds number $\mathrm{Re}_{\mathrm{h}}$ of 30000 . Preliminary measurements by LDV have shown that the boundary layer at the step corner presents a shape factor of 1.6, a momentum thickness of $1.63 \mathrm{~mm}$ and a displacement thickness of $2.62 \mathrm{~mm}$. At the flow separation, the Reynolds number $\left(\operatorname{Re}_{\theta}\right)$ scaled with the momentum thickness $\theta$ is equal to 1650 .
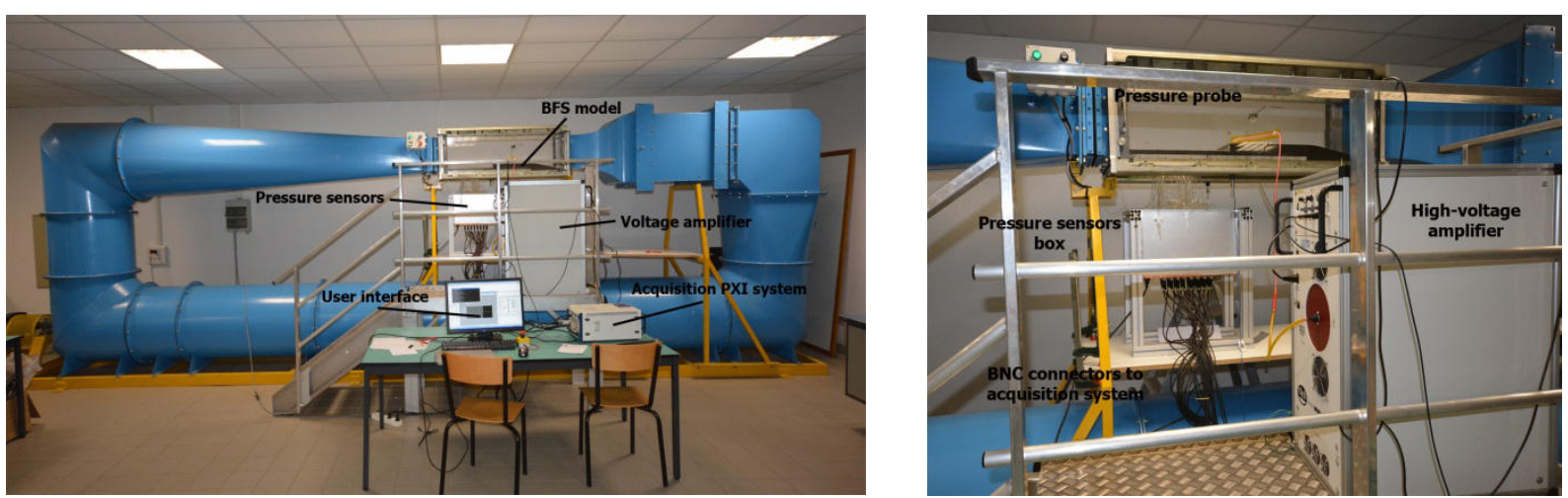

Figure 1 : Closed-loop wind-tunnel for backward facing step experiments. 
The step model is made of several pieces; in particular it has been designed in order to have a removable part. The removable part corresponds to the step geometry and is made of a 3-mm thick machined PMMA piece (Figure 2). So, the model can be used as dielectric barrier directly. The electrode arrangement is introduced in Figure 2. The air-exposed and the grounded electrode have a length of 15 and $10 \mathrm{~mm}$ respectively and the gap between them is fixed at $2 \mathrm{~mm}$. By construction, the electrode protuberance into the flow is limited to the electrode thickness (i.e. $60 \mu \mathrm{m}$ ) and then the initial turbulent boundary layer characteristics are not modified by the actuator.
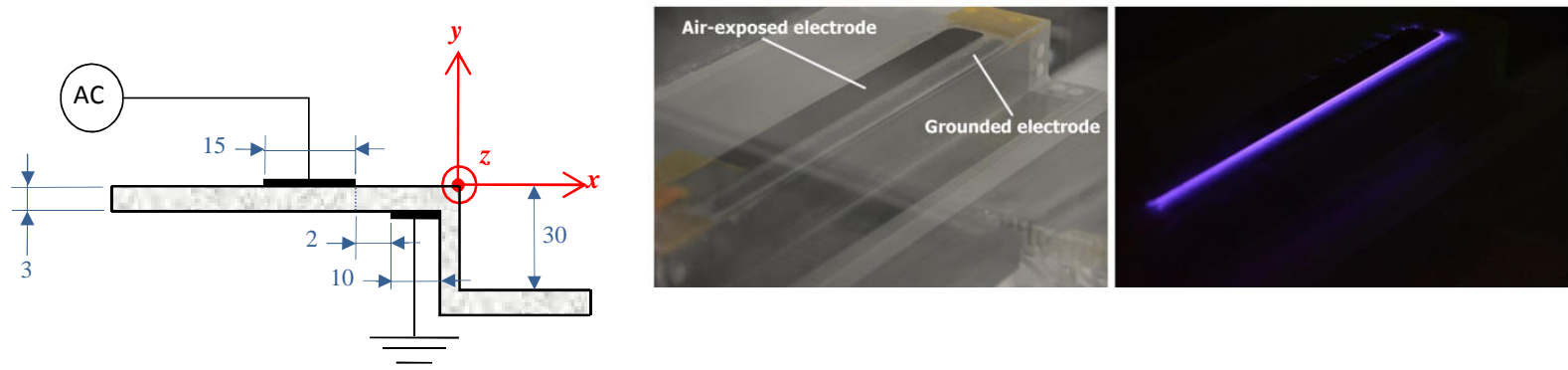

Figure 2 : Sketch of the DBD plasma actuator with details on electrode arrangement and co-ordinate system used in the paper and photos of the plasma actuator and the first pressure taps.

The actuator is a Dielectric Barrier Discharge (DBD) plasma actuator, a discharge well-known to produce a thin wall jet due to the electrohydrodynamic body force caused by the momentum exchanges between neutral and charged particles colliding in a weakly-ionized environment $[2,3,4$, 5]. Here the applied voltage is between 12 and $21 \mathrm{kV}$ in amplitude while the driving AC frequency is $2000 \mathrm{~Hz}$. In order to reach a frequency range for which the flow is receptive, the input signal is modulated by burst with variable duty-cycle. The low voltage electrical signal is produced by a generator card (PXI-5402), this low voltage being amplified with a scale factor of 3/3000 V (Trek 30/40) before supplying the top electrode. The parameters space of the optimization problem concern the voltage amplitude, the burst frequency and the duty-cycle of the electrical signal producing the surface discharge (Figure 3). In these conditions, it is well known that the mean amplitude of the produced flow increase with the voltage amplitude. Here, at the best the maximum velocity produced by the discharge does not exceed $4 \mathrm{~m} / \mathrm{s}$ (velocity ratio $\mathrm{U}_{\mathrm{DBD}} / \mathrm{U}_{0}=0.26$ ). The motivation for changing the burst frequency is that the mean flow and the vortex organization are very sensitive to periodic excitations at frequency matching with the preferred mode of the shear layer or its subharmonics [3536]. The last design variable is the duty-cycle. High duty-cycle value means that the produced flow approaches steady flow conditions (i.e., small fluctuation amplitude) while low duty-cycle will emphasize the amplitude of the fluctuations but in this case the mean produced flow velocity is small. Then, for flow control perspectives, a balance has to be found for producing a 'high' mean flow and one having 'high' fluctuation amplitude. All of these three parameters have strong influence on the performance of the control, this motivating for optimization studies using advanced, but model free, algorithms such as evolutionary algorithms. 


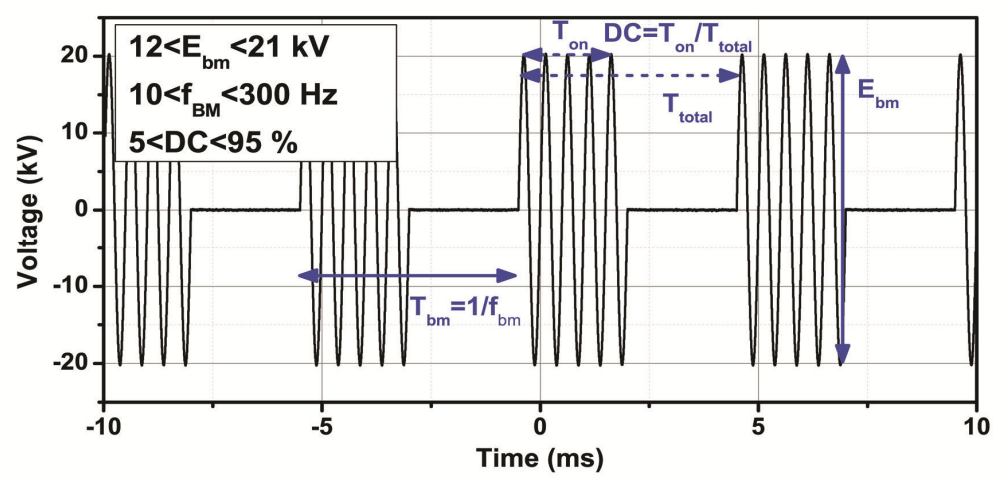

Figure 3 : Applied waveform and identification of the three design variables of the optimization problem.

The bottom part of the step model is equipped with 32 unsteady wall pressure sensors in its central plane (Figure 4a). These sensors are based on piezoelectric technology and they have a bandwidth of $2 \mathrm{kHz}$ for a maximum pressure of $250 \mathrm{~Pa}$. The output voltage signals of the pressure sensors are recorded by PXI hardware $(2.16 \mathrm{GHz}$ dual core) with 32 channels acquisition card (PXI6259). A LabVIEW code couples the acquisition and signal generator cards. The measures consist in recording the pressure fluctuation amplitudes at each sensor location and in computing the wall pressure fluctuation coefficients $\mathrm{C}_{\mathrm{P}^{\prime}}$. Then, the mean reattachment point is estimated by the position of the pressure fluctuation peak along the stream wise direction on the wall as indicated in literature [37]. The wall pressure fluctuation coefficients are computed in coefficient form as it follows:

$$
\mathrm{C}_{\mathrm{P}^{\prime}}=\frac{\sqrt{\frac{1}{n}\left[\left(P_{1}-P_{0}\right)+\left(P_{2}-P_{0}\right)+\cdots+\left(P_{n}-P_{0}\right)\right]}}{0.5 \rho U_{0}^{2}}
$$

where $\mathrm{P}_{0}$ and $\mathrm{U}_{0}$ refers to the reference value of the static pressure and streamwise freestream velocity, respectively. The number of data sample $\mathrm{n}$ is equal to 5000 at recording rate of $1000 \mathrm{~Hz}$.

An illustration of the $\mathrm{C}_{\mathrm{P}}$, distribution along the bottom wall is proposed in Figure $4 \mathrm{~b}$. The wall pressure fluctuation coefficient increases from the separation up to the reattachment where it reaches its maximal value. The control using a linear surface plasma systematically increases the amplitude of the pressure fluctuations at the wall and it reduces the reattachment location. Depending on the applied burst frequency, different amplitude in the wall pressure fluctuation coefficients can be observed. The reattachment is determined by the position of the pressure fluctuation peak. Here, a LabVIEW code is designed to autonomously determine this location, the objective function of the optimization problem. The error in the $X_{R}$ evaluation can be due by the pressure sensor but it main part comes from the spatial resolution of the wall sensors. In the present investigation, the maximum error on $X_{R}$ is $\pm 0.15 h$.

A custom made LabVIEW code manages the acquisition of the pressure sensors, the estimation of the objective functions, the electric command of the actuator and it makes the interface between the wind-tunnel and the optimizer. The interface between the optimizer and the wind-tunnel is done via DOS command under LabVIEW and the input/output interactions are managed through ASCII files 


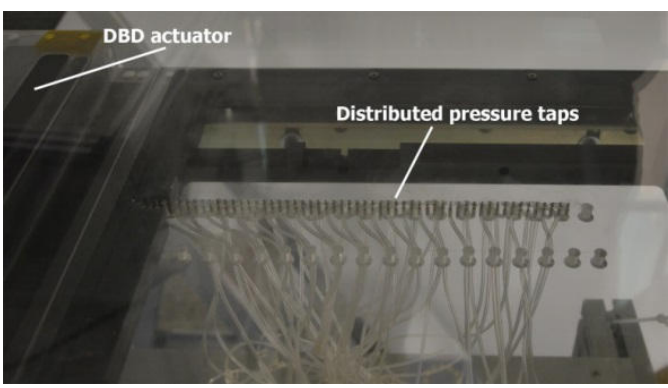

(a)

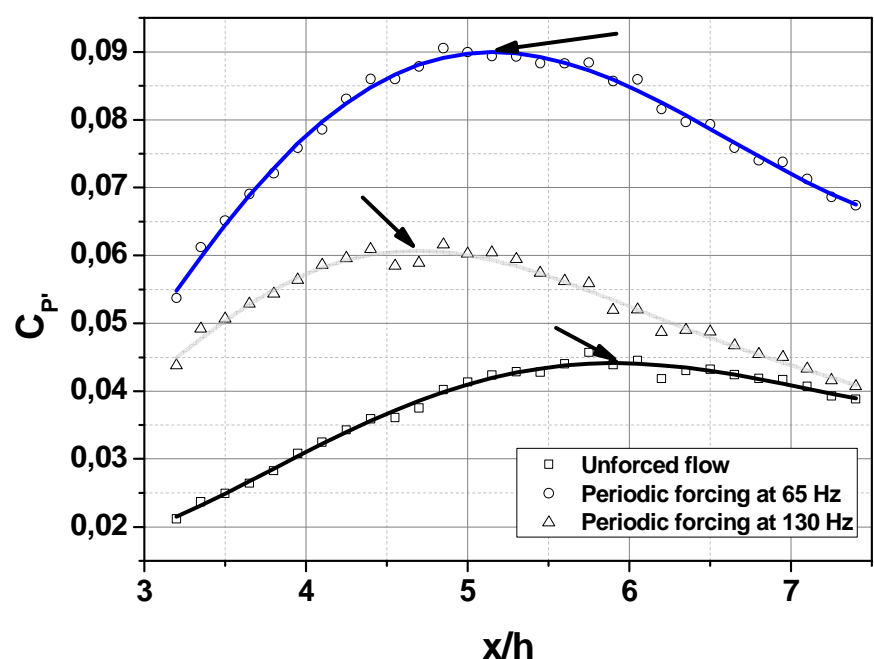

(b)

Figure 4 : View of the pressure taps along the bottom wall (a) and illustration of the pressure fluctuation coefficients in the streamwise direction for natural and controlled flows (b). The black arrows correspond to the estimated reattachment locations.

In a final experimental campaign, time-resolved particle image velocimetry is performed for the optimal control parameters. The flow downstream of the BFS is measured by a fast PIV system composed by a high-speed camera (Photron, APX-RS), a single head Nd:YLF high-speed laser with dual oscillator (Quantronix, Darwin-Duo), a triggering unit (EG, R\&D Vision) and a PC running Davis V8.2 software (Lavision). The laser (laser sheet of $1 \mathrm{~mm}$ ) is placed above the BFS at mid-span of the model to avoid lateral regions possibly affected by the boundary layer developing on the side wall of the tunnel. The camera is operated at 2000 full frames per second at $1024 \times 1024$ pixels $^{2}$. The flow is seeded with atomized oil (Ondina 15, Shell) resulting in $0.5 \mu \mathrm{m}$ trackers. Double-frame acquisitions are performed with frames separated by a time delay of $90 \mu \mathrm{s}$. The two velocity components are computed using a cross-correlation algorithm with adaptive multipass, interrogation windows of $64 \times 64$ to $16 \times 16$ pixels and an overlap set to $50 \%$. This results in a spatial resolution of $4.5 \times 4.5 \mathrm{~mm}^{2}$ for the vector fields. The maximum seeding particle displacement is approximately 8 pixels with a sub-pixel error of \pm 0.1 pixel, that corresponds to a minimal accuracy of $\pm 0.2 \mathrm{~m} / \mathrm{s}$. Flow sequences of 7.5 seconds are recorded. Acquisition starts 10 seconds after the plasma has been turned on, thus the transient regime is discarded from the analysis. The acquisition time is sufficiently long then all the time-averaged quantities (first and second order) have converged to their steady state values (variations lower than 5\%).

\section{The MOGA evolutionary optimizer}

Optimization methods define a set of tools and techniques that help engineers to solve complex problems optimizing an objective function while fulfilling a set of constraints [38]. This type of problem consists in maximizing or minimizing an objective function $f$ depending on $\mathrm{N}_{x}$ design variables varying over a bounded search space. There are different types of numerical algorithms for the solution of such optimization problem. Among the optimization methods available, the present investigation focuses on genetic algorithm approach, a stochastic method having demonstrated its robustness in complex problems where the objective function $\mathrm{f}$ is totally unknown. The solution is a unique or a set of individuals that have been selected by combination of selections, crossovers and mutations. 
Standard procedure starts with a randomly generated population (also called generation), including a predefined number of individuals. Each individual (made of three design variables, namely the voltage amplitude, burst frequency and duty-cycle) is evaluated by applying the corresponding signal command to the plasma discharge (see Figure 3). The objective is to minimize the function $X_{R}$, this function being evaluated by the wall pressure coefficients. The evaluation of one individual lasts 15 seconds, this time including 5 seconds for achieving a stable control, 5 seconds of data sampling of the wall pressure fluctuations and 5 seconds to let the flow fully relax. The code computes the wall pressure fluctuation coefficients and it determines the fitness function $X_{R}$ of the evaluated individual during the flow relaxation. The same procedure is reproduced for all individuals of one generation. At the end of the evaluation of a population, individuals are selected, crossed-over or mutated to generate new offspring to be tested. The iterative process finish when the stopping criterion is reached (it could be accuracy, time, number of iterations, etc...). The stopping criterion applied in this research is maximal time duration of one hour, this corresponding to a total of 12 generations. The basic operators, which manage the genetic algorithm, are the selection, the crossover and the mutation. The selection operator takes care of selecting the best performing individuals of each population to enable other operators to produce new offspring. It is the most basic operator and usually the first one to be applied. The most usual selection techniques are roulette wheel, ranking selection, and elitism, among others. Here a ranking selection is applied. Crossover is taking two selected individuals and it combines them to create a new offspring. Several techniques can be used to determine the point where the genetic information is split to add the genetic information from the second individual. The most standard techniques for crossover include one-point crossover (as in the present work), two-point crossover, where one or two splitting points are defined to combine the information from the individuals, uniform crossover, where a random combination of bits is used or arithmetic crossover, where an arithmetic operation defines the combination strategy. Finally, the mutation operator creates new offspring modifying the information contained in one selected individual. Bit Inversion and order changing are usual mutation techniques for binary encoding, while the addition of small quantities is typical of real-value encoding as used in the present work.

The selected optimizer used in this work is the Multi-Objective Genetic Algorithm (MOGA) module in Robust Multi-objective Optimization Platform (RMOP) developed at CIMNE. Details of RMOP can be found in references [39, 40, 41, 42]. This optimization platform enables the analysis of multi-objective problems using the hybridized techniques that combine Pareto-game and Nash-games. The basic operators used are ranking selection (tournament selection where the ranked, i.e. best fitted, individuals are stored in a buffer) and single-point crossover (crossover probability of 0.9). The mutation is the one for a real-value encoding. Here, the mutation probability is a function of the number of design variables; it is defined as $1 / 3$ because three designs variables are considered. Nevertheless, in this work only the subset of tools corresponding to single objective optimization problems has been used and this simplified use of the optimization code is not using the parallel computing capabilities useful for CFD simulations.

\section{Experimental results}

The results of this investigation are divided regarding the use of an autonomous parametric study or an optimization related to the use of a GA code. In a final part, the flow modifications caused by the discharge are presented when the plasma actuator is operated at its optimal electrical conditions. 


\section{IV-1 Inverse reconstruction problems}

At our best knowledge, the procedure of coupling experimental sensors and actuators with a numerical optimization code, as an analogic solver, has never been performed. So, as a preliminary stage, the approach has been carefully validated in order to ensure that the methodology is able to solve complex optimization problems. It has been decided to test the coupled experimental-numerical optimization procedure with the following inverse problem:

Problem: A single objective problem to recover a prescribed reattachment length $X_{R}$. This prescribed value is defined from the results from Sujar-Garrido et al. [27], a study using the same BFS configuration and actuator, but measuring the flow response to actuation by stereoscopic PIV. In this paper, a parametric study on the influence of the burst frequency has been conducted with a coarse resolution for the investigated frequency range due to the inherent difficulties of stereo-PIV, however a clear minimization of the recirculating zone has been observed at a forcing frequency of $125 \mathrm{~Hz}$. The minimal reattachment obtained at this frequency is $4.7 \mathrm{~h}$. For this inverse problem, the prescribed reattachment length $X_{R}$, is fixed at $4.7 \mathrm{~h}$ in agreement with [27].

The objective is to minimize

$$
f_{i}\left(\boldsymbol{x}_{1}, \boldsymbol{x}_{2}, \boldsymbol{x}_{\mathbf{3}}\right)=f_{e i}\left(\boldsymbol{x}_{\mathbf{1}}, \boldsymbol{x}_{2}, \boldsymbol{x}_{\mathbf{3}}\right)-f_{p i}\left(\boldsymbol{x}_{1}, \boldsymbol{x}_{2}, \boldsymbol{x}_{\mathbf{3}}\right), \quad i=1,2
$$

The values $f_{i}\left(\boldsymbol{x}_{1}, \boldsymbol{x}_{2}, \boldsymbol{x}_{3}\right)$ are the fitness functions of the inverse problem. The values $f_{p i}\left(\boldsymbol{x}_{1}, \boldsymbol{x}_{2}, \boldsymbol{x}_{3}\right)$ and $f_{e i}\left(\boldsymbol{x}_{1}, \boldsymbol{x}_{2}, \boldsymbol{x}_{3}\right)$ are the prescribed value and the evaluated value for the objective function $\mathrm{X}_{\mathrm{R}}$.

The definition of the vector search space is made of three design variables $\left(\boldsymbol{x}_{1}, \boldsymbol{x}_{2}, \boldsymbol{x}_{\mathbf{3}}\right)$ :

- $\quad x_{1}$ : Voltage amplitude (V); $\mathrm{V} \in \mathbb{N}, 12 \leq \mathrm{V} \leq 21$

- $\quad \boldsymbol{x}_{2}$ : Burst Frequency $\left(f_{b m}\right.$, in $\left.\mathrm{Hz}\right) ; f_{b m} \in \mathbb{N}, 10 \leq f_{b m} \leq 300$

- $\quad x_{3}$ : Duty Cycle (DC, in \%); DC $\in \mathbb{N}, 5 \leq \mathrm{DC} \leq 95$

This inverse problem is solved as traditional reconstruction problems with the main characteristic that the final solution is known from the literature for given values of the triplet $\boldsymbol{x}_{1}, \boldsymbol{x}_{2}, \boldsymbol{x}_{3}$. Thus, the recovery of the prescribed solution validates the operability of the optimization procedure. Each generation is composed of ten individuals, with a random selection for the first generation.

To illustrate the GA code process and its convergence, the evaluation of the best individual of one generation are shown in Figure 5 for all tested generations. In Figure 6 is shown the deviation to zero for the ten individuals of the first generation. This generation is randomly selected as evidenced by the wide disparity in the applied voltage, frequency and duty-cycle values. At the best the deviation reaches 0.4 . From the fourth generation, the voltage amplitude already converges to its maximal possible value (i.e. $21 \mathrm{kV}$ here). After four additional generations, the three design variables converge to their final values. At the end of the procedure, the GAs optimizer coupled with the plasma actuators and the 32 unsteady sensors captures the solution of the inverse problem. Most of the final population presents evaluated values which compared to the prescribed value leads to a deviation of the objective function equal to zero. Some individuals of the final population presents an objective function lower than zero, indicating that larger recirculation reduction is achieved in the present investigation by comparison with results published in [27]. In fact, the driven frequency used here is $2000 \mathrm{~Hz}$ when only $1000 \mathrm{~Hz}$ was used in [27]. This larger driven frequency should increase the mean flow produced 
by the actuator as described in the literature [5]. One has also to remind that the error in $\mathrm{X}_{\mathrm{R}}$ estimation is of about $0.15 \mathrm{~h}$ in the present investigation. Regarding the design variables, the three parameters that recover the optimal individual are:

- Voltage amplitude was $24 \mathrm{kV}$ in [27] and the obtained values is $21 \mathrm{kV}$,

- Burst Frequency was $125 \mathrm{~Hz}$ in [27], and the obtained one is $126 \mathrm{~Hz}$,

- and finally the duty-cycle was 50\% in [27], when the obtained one is $60 \%$.

The fast convergence of the GA code to solve the inverse problem solution validates the experimental-numerical methodology.

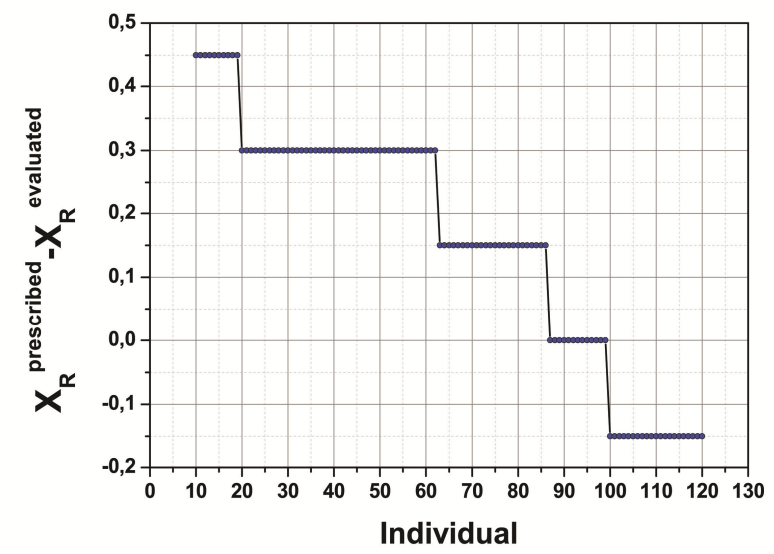

Figure 5 : Convergence history for the inverse problem minimizing the reattachment length.
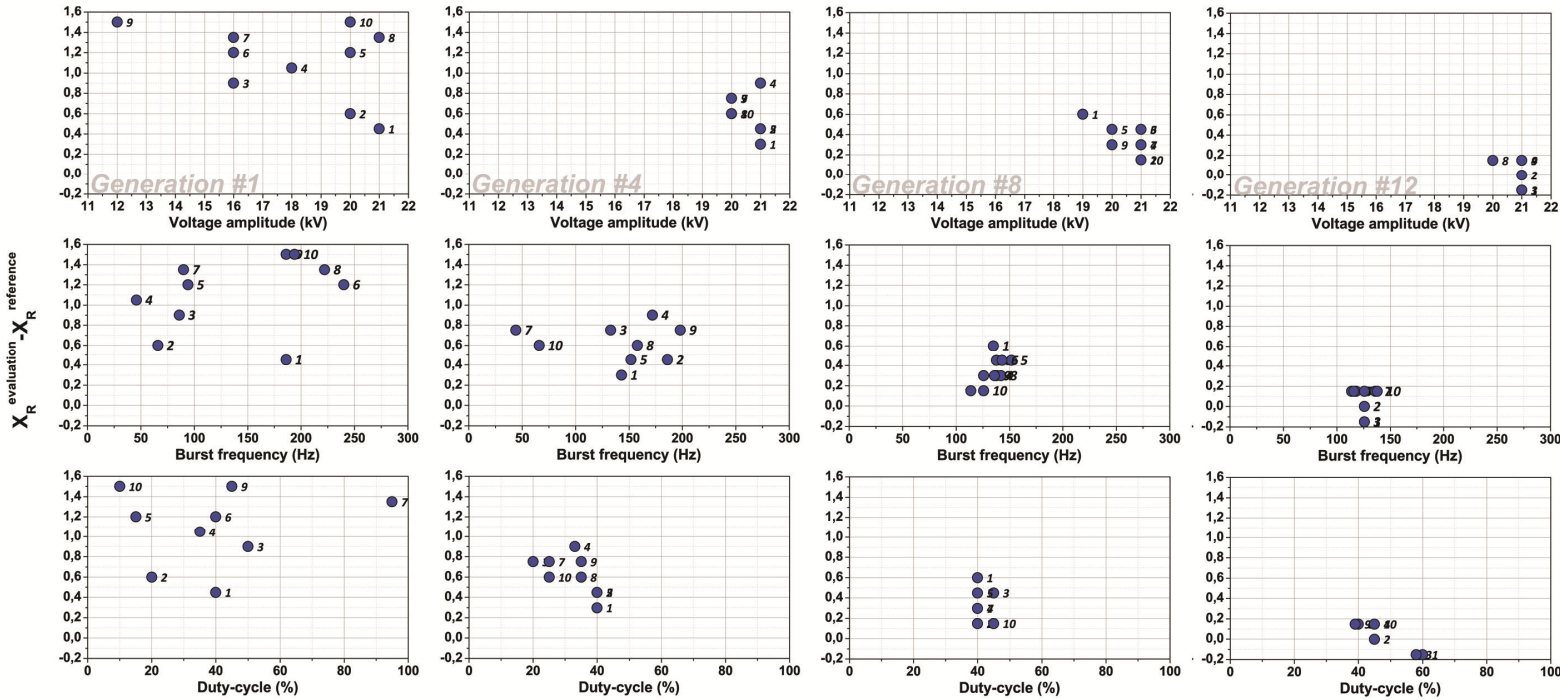

Figure 6 : Evaluation of the individuals of a generation for the initial random generation of the inverse problem, generation \#4, generation \#8 and the final generation \#12.

\section{IV-2 Optimization with the MOGA optimizer}

The primary objective of this paper is to test GA approach in order to minimize the recirculation downstream of a BFS. The objective function is then the reattachment length $X_{R}$ and the optimization problem is defined as:

Minimize 


$$
f_{o}(\overrightarrow{\boldsymbol{x}})=\min \left[X_{R}(\overrightarrow{\boldsymbol{x}})\right]
$$

where $\overrightarrow{\boldsymbol{x}}$ is the vector containing the three design variables of the DBD plasma actuator.

The optimization problem, equation (3), is defined as a single-objective minimization problem, with the following definition of the lower-upper bounds search space of the three design parameters:

- Voltage amplitude (V, in $\mathrm{kV}) ; \mathrm{V} \in \mathbb{N}, 12 \leq \mathrm{V} \leq 20$

- Burst Frequency $\left(f_{b m}\right.$, in $\left.\mathrm{Hz}\right) ; f_{b m} \in \mathbb{N}, 10 \leq f_{b m} \leq 300$

- Duty-Cycle (DC, in \%); DC $\in \mathbb{N}, 5 \leq \mathrm{DC} \leq 95$

To illustrate the GA code process and its convergence, the evaluation of the individuals of different generations are shown in Figure 7,8 and 9. In these figures is shown the evolution of objective function $X_{R}$ when the design variables are simultaneously optimized. Since the first generation is randomly defined, the design variables cover the whole search space. However, the GA approach finds the optimal voltage amplitude in only a few generations (Figure 7). The mean reattachment position varies from $6.2 \mathrm{~h}$ down to $4.55 \mathrm{~h}$. The fast convergence rate is caused by the plasma actuator itself. Indeed, the increase in mean flow velocity produced by the actuator with the voltage amplitude can be fitted by a second order polynomial. Increasing the voltage amplitude has a strong influence on the reduction of the recirculation zone. But too high amplitude promotes a filamentary discharge, a plasma regime less effective to produce EHD effects. This explains the convergence to an optimized value of $20 \mathrm{kV}$. The evolution of the code optimizing the burst frequency is shown in Figure 8. The convergence rate is lower for this variable indeed the mutation of the individuals slows the convergence rate but it also insures that the best solution is found, this providing the necessary robustness to the optimisation algorithms. The first three generation converges to values about $175 \mathrm{~Hz}$. In the fourth generation, one of the individual (\#4) mutated and it finds a better minimization of $X_{R}$. Then, the variable $x_{2}$ of the individual converges to a new optimum. The evolution of the duty-cycle is shown in Figure 9. As for the burst frequency, the GA code needs several generations to converge to a duty-cycle between 40 and $70 \%$. 

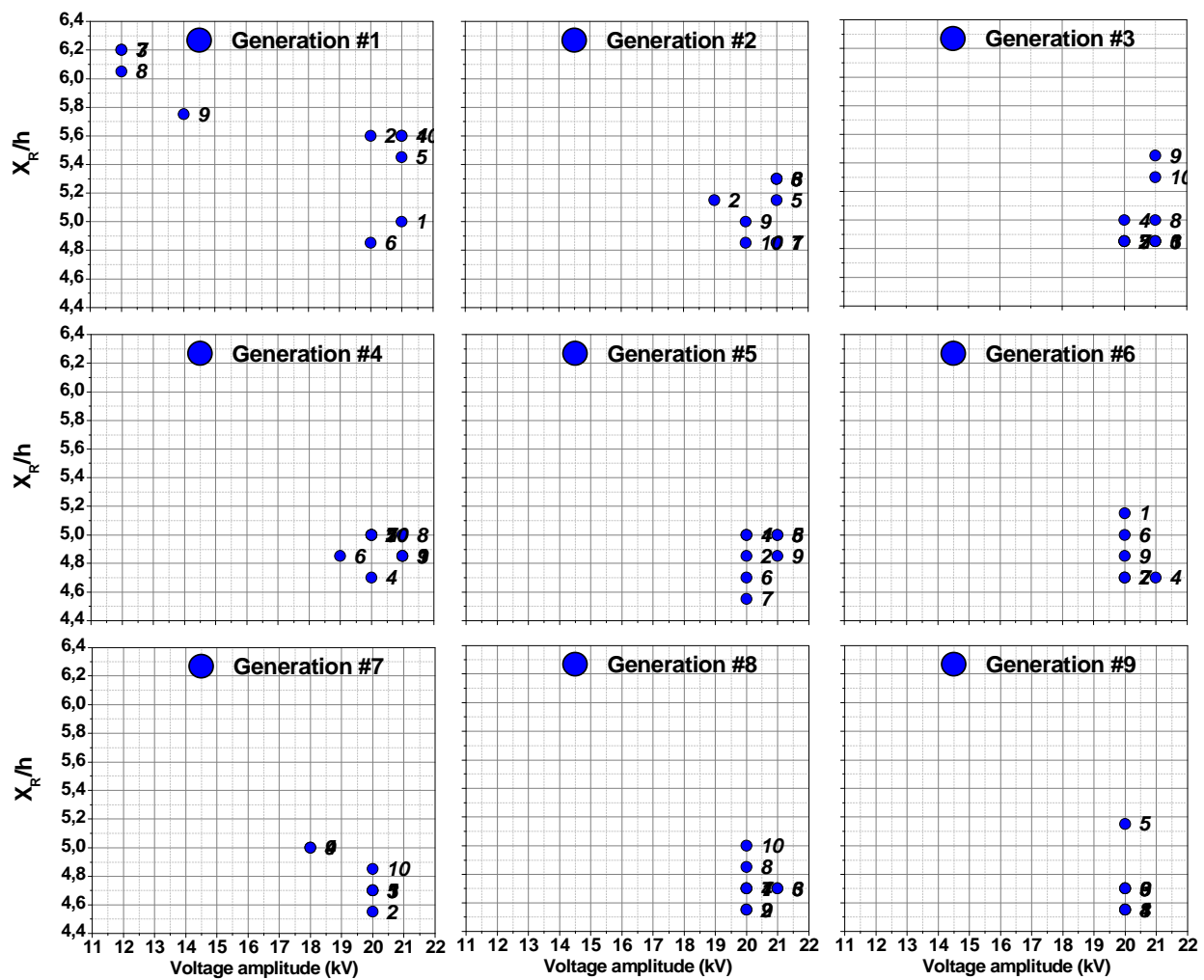

Figure 7 : Evolution of the objective function $X_{R}$ for the first nine generations when the voltage amplitude (design parameter $x_{1}$ ) is varied (numbers relate to the identification number of the individuals).
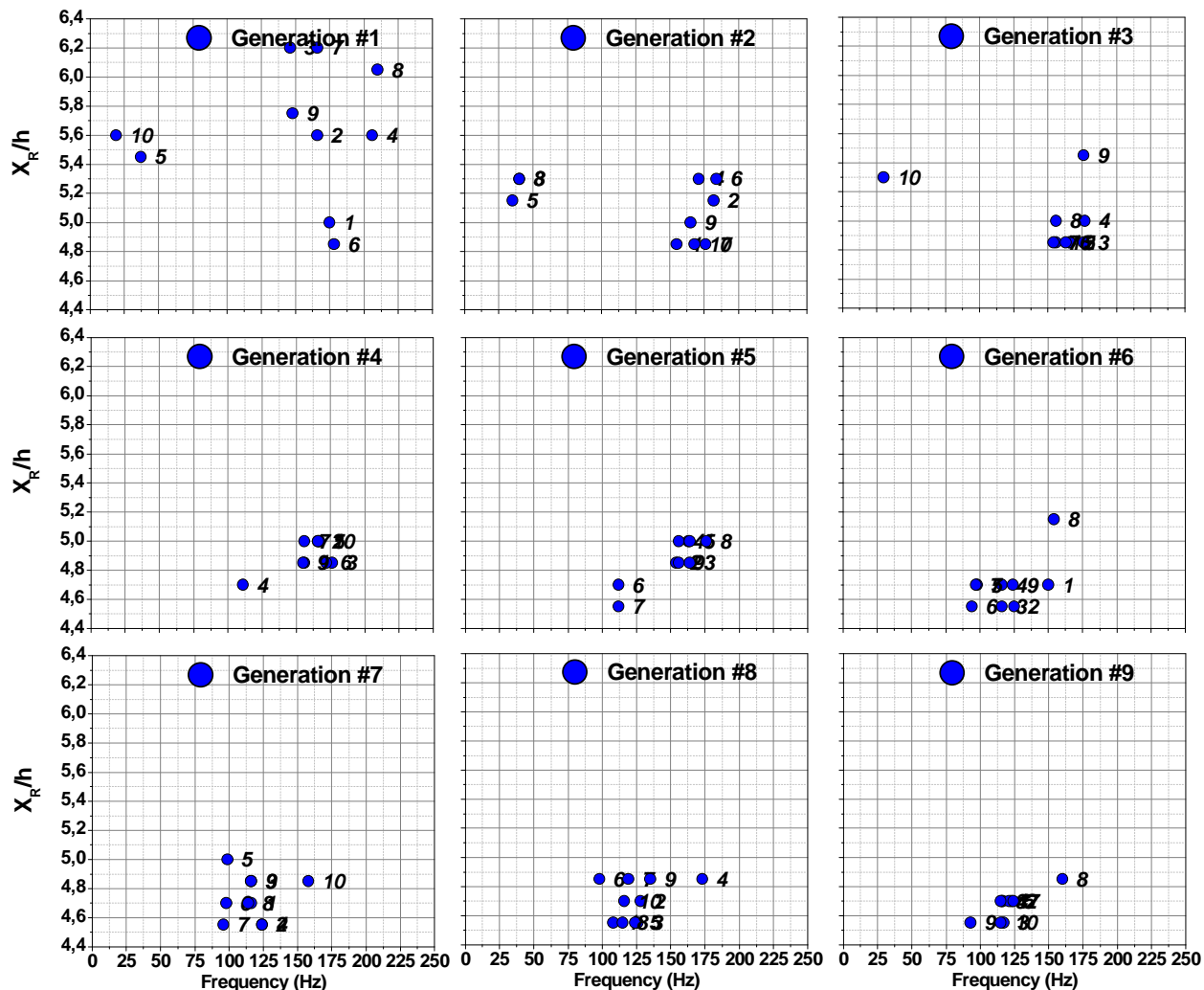

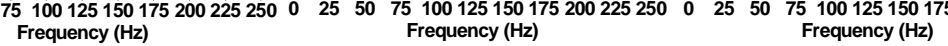

Figure 8 : Evolution of the objective function $X_{R}$ for the first nine generations when the burst frequency (design parameter $\mathrm{x}_{2}$ ) is varied (numbers relate to the identification number of the individuals). 


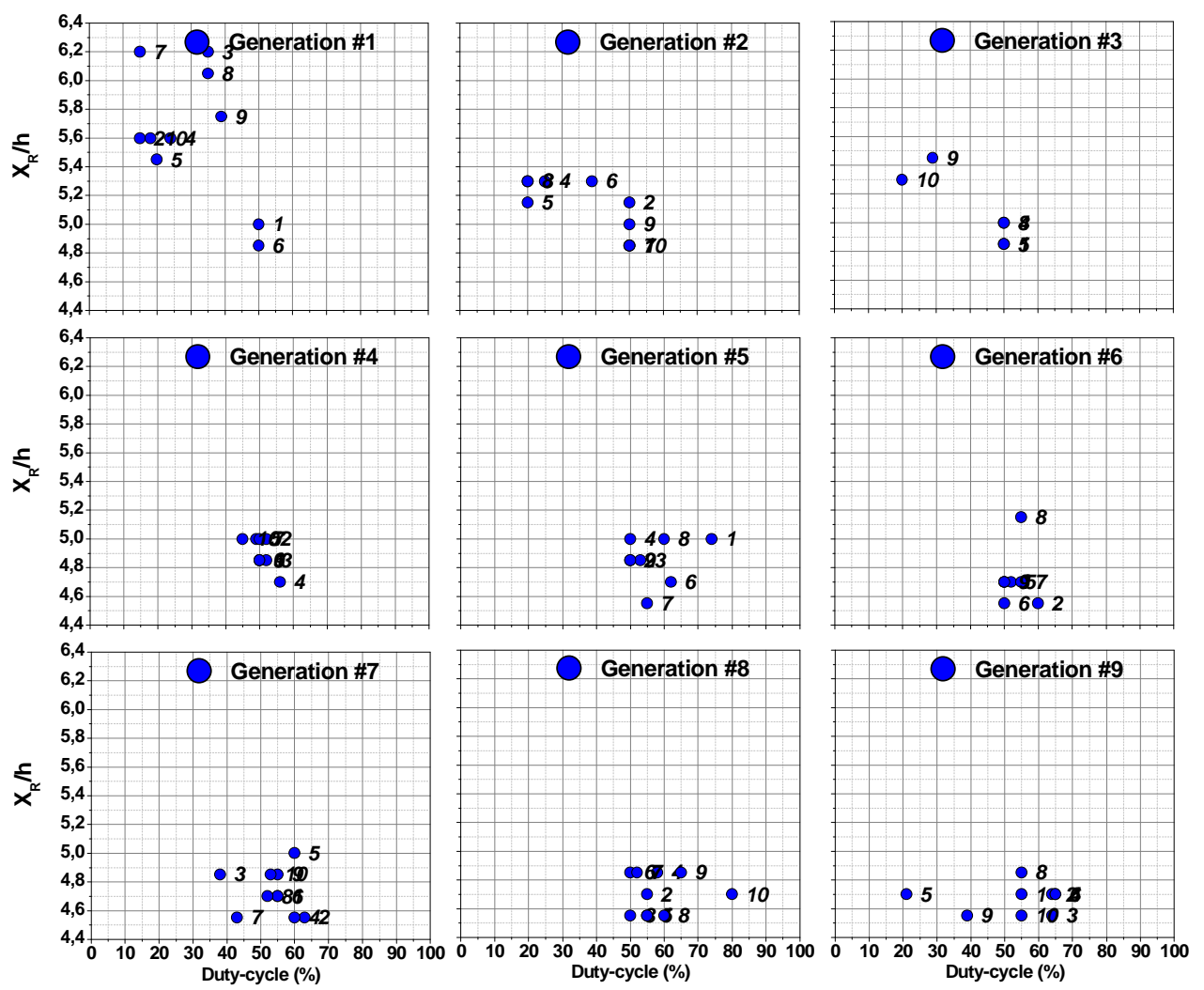

Figure 9 : Evolution of the objective function $X_{R}$ for the first nine generations when the duty-cycle (design parameter $x_{3}$ ) is varied (numbers relate to the identification number of the individuals).

The results for the final generation as well as the convergence of the individuals are plotted in Figure 10. The individuals of this generation correspond to the optimized solutions. For the worst individuals, a mean reattachment at $6.5 \mathrm{~h}$ has been measured. However, the GA code coupled with the plasma discharge reduces the recirculation by $30 \%$, the reattachment point moving up to $4.55 \mathrm{~h}$. This significant reduction in the mean flow reattachment is observed for the following three design variables:

- Voltage amplitude of $20 \mathrm{kV}$

- Burst Frequency of about $125 \pm 5 \mathrm{~Hz}$

- Duty-cycle of about 50-80\%

The gain in $X_{R}$ is precisely the one already obtained in [26] or [27]. However, here the optimization is performed in only one hour when PIV measurements as done in [27] take several months for processing the data. The convergence plot confirms that the GA code quickly evolves to the optimized solution for this simple flow control application.

The optimization by GA code conduces to a forcing mode largely documented in the literature for other types of flow control devices than the surface plasma discharge used in this investigation. The physical best forcing frequency expressed in normalized form leads to a Strouhal number $\mathrm{St}_{\mathrm{h}}=0.24$. For instance, Chun and Sung identified the optimal local forcing by loud speaker at $\mathrm{St}_{\mathrm{h}}=0.27$ in [43] $\left(\operatorname{Re}_{\theta}=1470\right)$. Periodic excitation at this frequency corresponds to the step mode of instability of the separated shear layer as it is detailed in [17]. Indeed, at the initial stage of its development, the shear layer is composed of vortices separated by a short wavelength. The shear layer develops and grows by vortex pairing up to a certain spatial location.t this location, the vortex amalgamation ends leading to a 
final shedding frequency (as the preferred mode in jet flow) that have been called step mode in [17]. The natural instability frequency of the reattaching shear layer also scales with the momentum thickness $\theta$. The Strouhal numbers $\mathrm{St}_{\theta}$ for the best candidate obtained from the GA optimizer is 0.013. The case of forcing at $\mathrm{St}_{\theta}=0.013$ is well known to be related to the shear layer mode of instability [17]. Here, the GA code reveals that the most effective forcing condition to minimize the recirculation corresponds to applying low amplitude perturbations at the fundamental frequency. However, it is widely admitted that the coherent flow structures of a mixing layer or a turbulent separated shear layer can be manipulated with periodic excitations that can promote vortex pairing by sub-harmonic forcing $[44,45]$. Here, the GA optimizer does not reveal effective control scenario by applying perturbations near a sub-harmonic of the most-amplified frequency, the best forcing conditions being related to direct interactions with the shear layer mode. Spite of having not identified a new control scenario, the present validation of the code suggests that it can be applied to various flow configurations; this avoiding exhaustive and long parametric study when multi-parameters have to be optimized. Furthermore, it is expected that new control scenario may emerge from the use of multi-input and multi-objective genetic algorithm approaches in complex experiments with multiple independent actuators. For instance, by using an appropriated input signal composed of fundamental and harmonics tuned in amplitude and phase, the contribution of vortex splitting, merging and collective aspects of a controlled separated shear flow can be experimentally optimized as it was numerically investigated in [46].
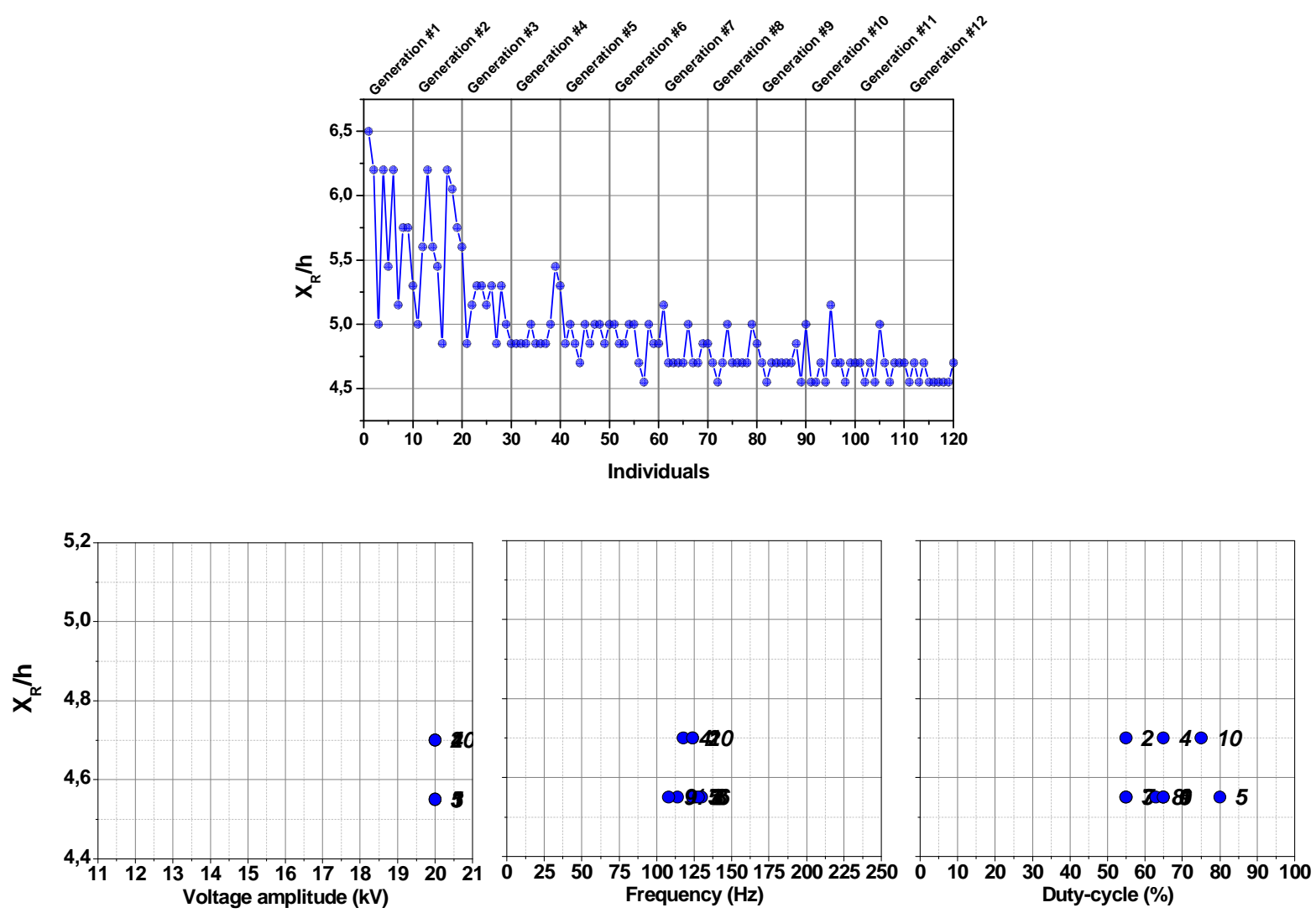

Figure 10 : Convergence of the GA optimizer (top) and fitness function of the final generation (\#12) according to the voltage amplitude, the burst frequency and the duty-cycle. 


\section{IV-3 Time-resolved measurement of the turbulent flow field downstream of the BFS}

The GA code has found the best forcing conditions that minimize the reattachment position. In a second step, a PIV experimental campaign has been conducted. The objective focused on the characterization of the natural BFS mean flow and flow when the best forcing condition identified from the GA optimizer are applied. A same fast PIV system is used for measuring the mean and dynamic characteristics of the flows.

\section{IV-3-1 Mean flow quantities}

The mean velocity field normalized by the freestream velocity $\mathrm{U}_{0}$ is plotted in Figure 11 for the natural flow case as well as the best forcing as defined from the GA code. The iso-contours or the representation by streamlines confirm that the actuation leads to a reduction of the recirculating bubbles. From these data, the location of the reattachment point is determined as the point where the velocity $\mathrm{U}$ is equal to zero at a distance of $0.05 \mathrm{~h}$ from the bottom wall (see Figure 12). The natural flow reattaches at $5.8 \mathrm{~h}$ downstream of the step wall. This value corroborates with the reattachment location extracted from the wall pressure fluctuations distribution. When the plasma discharge is operated at $20 \mathrm{kV}, 125 \mathrm{~Hz}$ and $60 \%$ of duty-cycle ( $\mathrm{St}_{\theta} \approx 0.013$ ), the mean flow reattaches at $4.6 \mathrm{~h}$. The flow measurements confirm the $20 \%$ reduction in the reattachment location due to the DBD actuator.
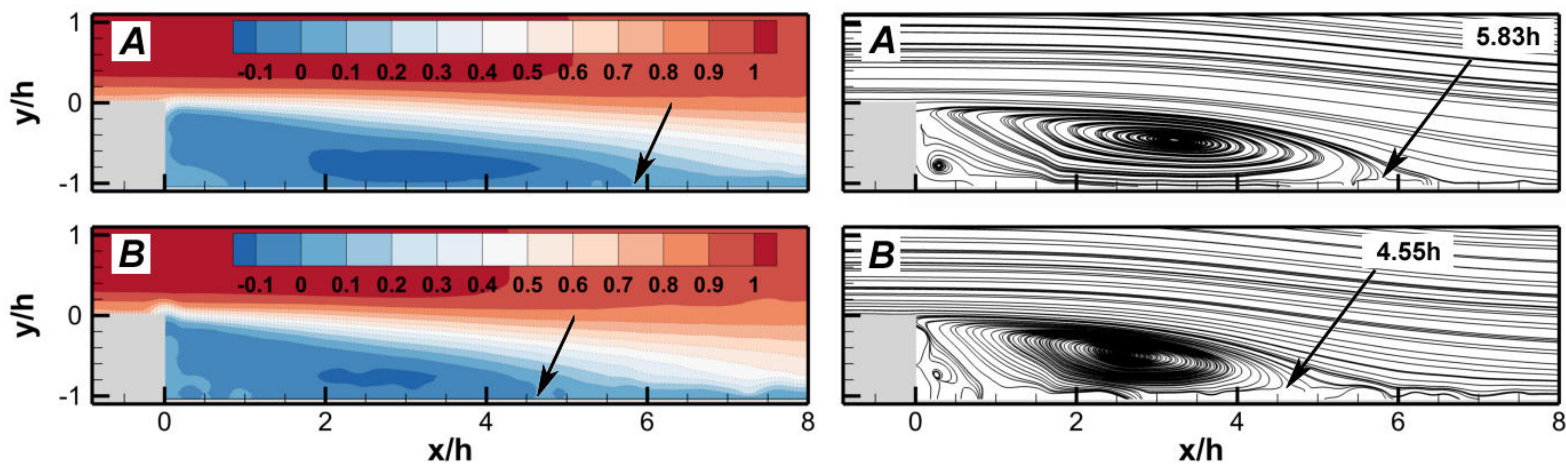

Figure 11: Mean velocity field $U$ normalized by $U_{0}$ (left plot), and the streamlines of the flow for the two optimal sets of design variables (right plot). The baseline flow is presented in (a), and plasma actuation at $\mathrm{St}_{\mathrm{h}}=0.23, \mathrm{St}_{\theta}=0.013$ in (b).
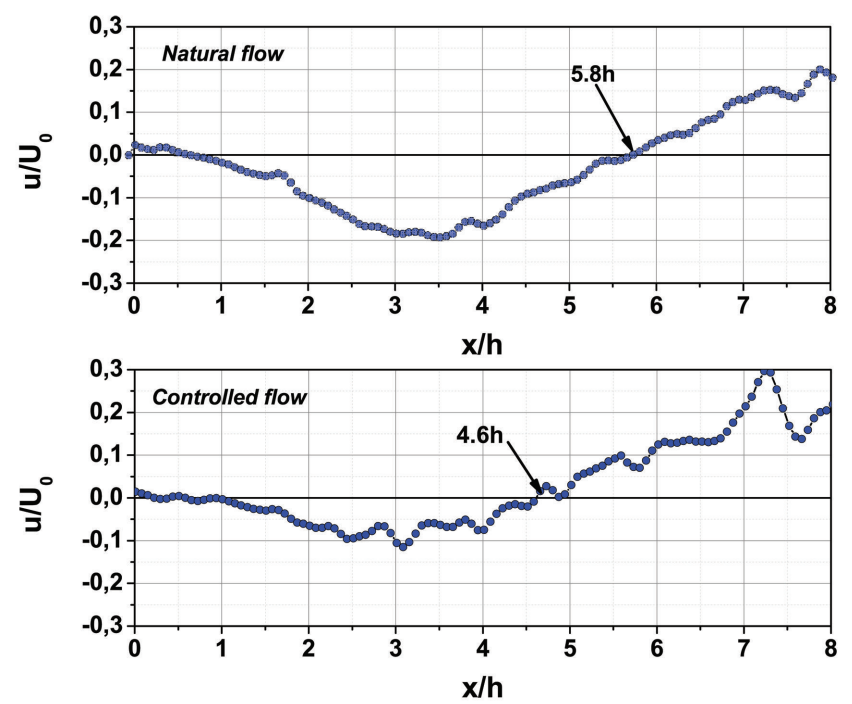

Figure 12 : Velocity extraction at $\mathrm{y} / \mathrm{h}=-0.95$ for determination of the mean reattachment location 
The influence of a periodic forcing at $\mathrm{St}_{\theta}=0.013$ is further demonstrated by inspecting the evolution of the vorticity thickness with downstream location $\mathrm{x} / \mathrm{h}$ (Figure 13). For the natural and controlled flows, the vorticity thickness linearly grows with the downstream distance in the earlier instants of the turbulent shear layer development (zone I in Figure 13). This figure demonstrates that the actuation has almost no influence on the shear layer development up to $\mathrm{x}=2.5 \mathrm{~h}-3 \mathrm{~h}$. The growing mechanism in the first instants is not clear at this stage, but it appears that the actuation does not modify the growth rate of the separated shear flow. The main difference for position $x>3 h$ is that the linear region stops at $\mathrm{x}=3.5 \mathrm{~h}$ for the natural flow and it is followed by a region where the thickness of the shear layer stabilizes (zone II in Figure 11a). This constant vorticity thickness should result from the completion of the merging process or because the vortices stop their development in size for any reason [44]. Beyond the reattachment point (end of zone II), the vorticity thickness increases again due to the lateral displacement of the reattaching region (zone III). With the plasma discharge, the shear layer stops its primary development at a shortened distance $(x=3 \mathrm{~h})$ compared to the natural flow, but the vorticity thickness still increases up to $\mathrm{x}=4.5 \mathrm{~h}$ (zone II). It is easily seen that the vorticity thickness increases at a slower rate than that in zone I. It can be assumed that zone II corresponds to a change in the growing mechanism of the fully developed shear layer compared to zone I. The amalgamation of adjacent vortices contributes to a larger spreading of the shear layer but the pairing of vortical flow structures is not the only mechanism to promote the divergence of a turbulent shear layer. For instance, a smaller spreading rate has been observed in the process of vortex splitting than in vortex merging [47]. Furthermore, the shear layer growing can be caused by the growth of eddies by fluid entrainment and not to the pairing mechanism as shown in Brown and Roshko [48]. Unfortunately, an analysis based only on mean flow quantities cannot reveal the real dynamic corresponding to the flow in zone II.

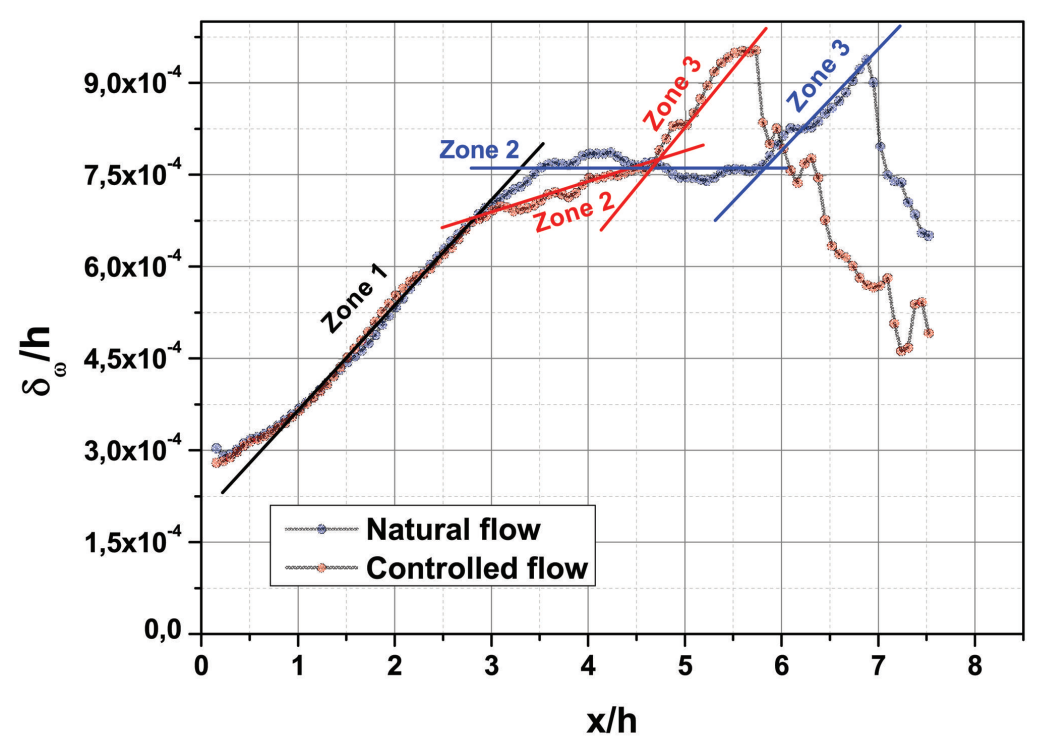

Figure 13 : Evolution of the vorticity thickness with the downstream distance from the step

\section{IV-3-2 Dynamics of the turbulent flow field}

To visualize the temporal evolution of the separated shear layer, the fluctuating velocity v' is extracted from the PIV fields at $\mathrm{y} / \mathrm{h}=0$ (Figure 14). In such a representation, a vortical flow structure presents a signature composed of an alternation of positive and negative v' velocity components. In all the plots, the convection of the vortical flow structures is clearly visible. The convection velocity, $\mathrm{U}_{\mathfrak{c}}$, is $0.55 \mathrm{U}_{0}$ for the natural flow and it slightly reduced to $0.5 \mathrm{U}_{0}$ when the plasma discharge is applied. The plasma 
discharge reinforces the amplitude of the velocity fluctuations. Indeed, the main result from this figure is the strong regularization of the vortical flow structures of the shear layer for a periodic forcing at the shear layer mode $\mathrm{St}_{\theta}=0.013$. By forcing the inlet flow conditions with small amplitude perturbations at $\mathrm{St}_{\theta}=0.013$, the flow quickly organizes in a periodic manner (from $\mathrm{x} / \mathrm{h}=1$ ) and it exhibits a strong periodization between $x / h=2$ and $x / h=4$. From Figure 14 , it seems that the frequency of the vortical signature reduces past the $\mathrm{x} / \mathrm{h}=4$ position. The flow dynamic imposed by the forcing conditions is further evidenced by the extraction line along the fluctuating velocity time history as proposed in Figure 15 for different streamwise positions. For the controlled case, the flow presents periodic oscillations at a frequency of about $125 \mathrm{~Hz}$ in the first instants of the shear layer development (i.e., $\mathrm{x}=2 \mathrm{~h}$ ). As compared with the natural flow, the periodic nature of the turbulent flow is more pronounced with the plasma discharge, but the natural flow also exhibits some regular flow pattern. However, at $x=2 h$ the frequency of the periodic structuration of the separated shear layer is reduced by the surface plasma discharge compared to the natural flow. The frequency of the natural flow is approximately twice the one for the controlled flow at this location. For measurements locations at $\mathrm{x}=3 \mathrm{~h}, 4 \mathrm{~h}$ and $5 \mathrm{~h}$, the surface plasma discharge imposed the dynamic of the entire shear layer with periodic oscillations having a frequency equals to the forcing frequency regardless of the investigated position. The natural flow presents a more complex behavior than for forcing at $\mathrm{St}_{\theta}=0.013$, the extraction lines confirm the presence of oscillations in the flow, but a constant wavelength cannot be easily defined. The present results indicate that the linear plasma discharge imposes the periodic oscillations and vortex shedding in the separated shear layer by forcing the shear layer mode.
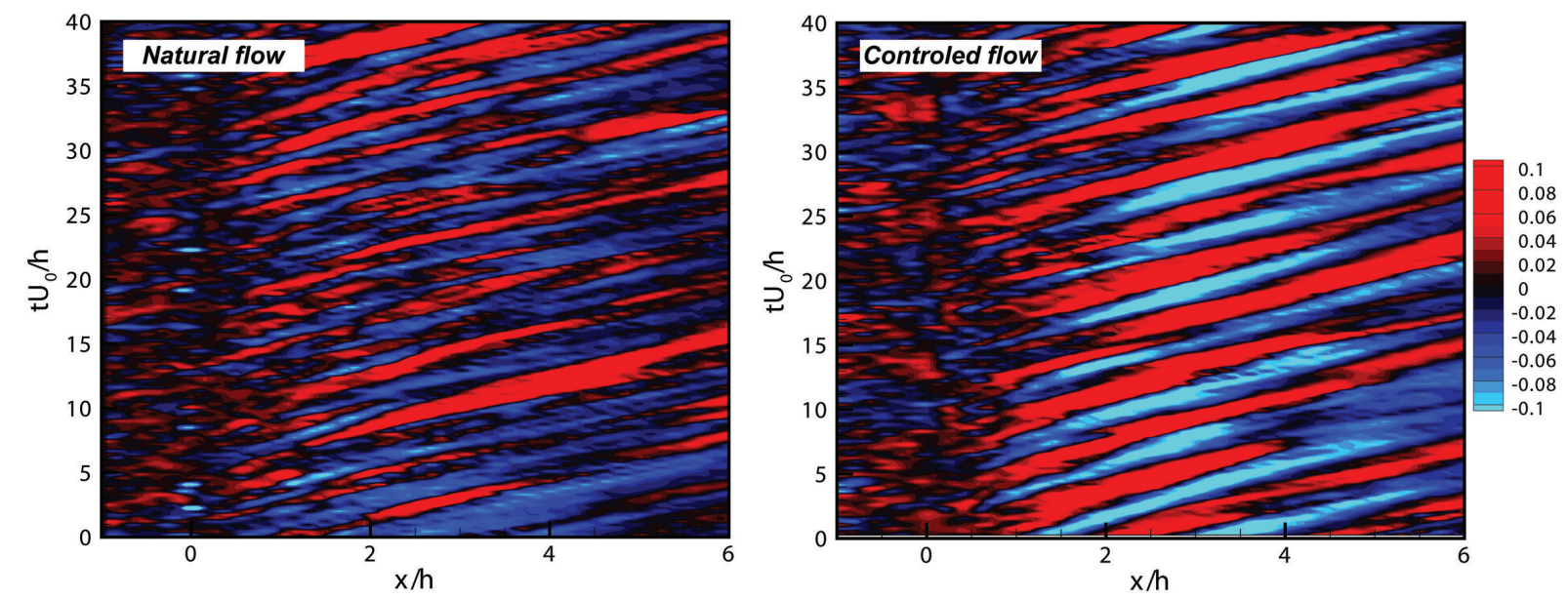

Figure 14 : Time evolution of the fluctuating velocity $v^{\prime}($ at $y / h=0)$ for the natural flow and periodic forcing at $\mathrm{St}_{\theta}=0.013$. 

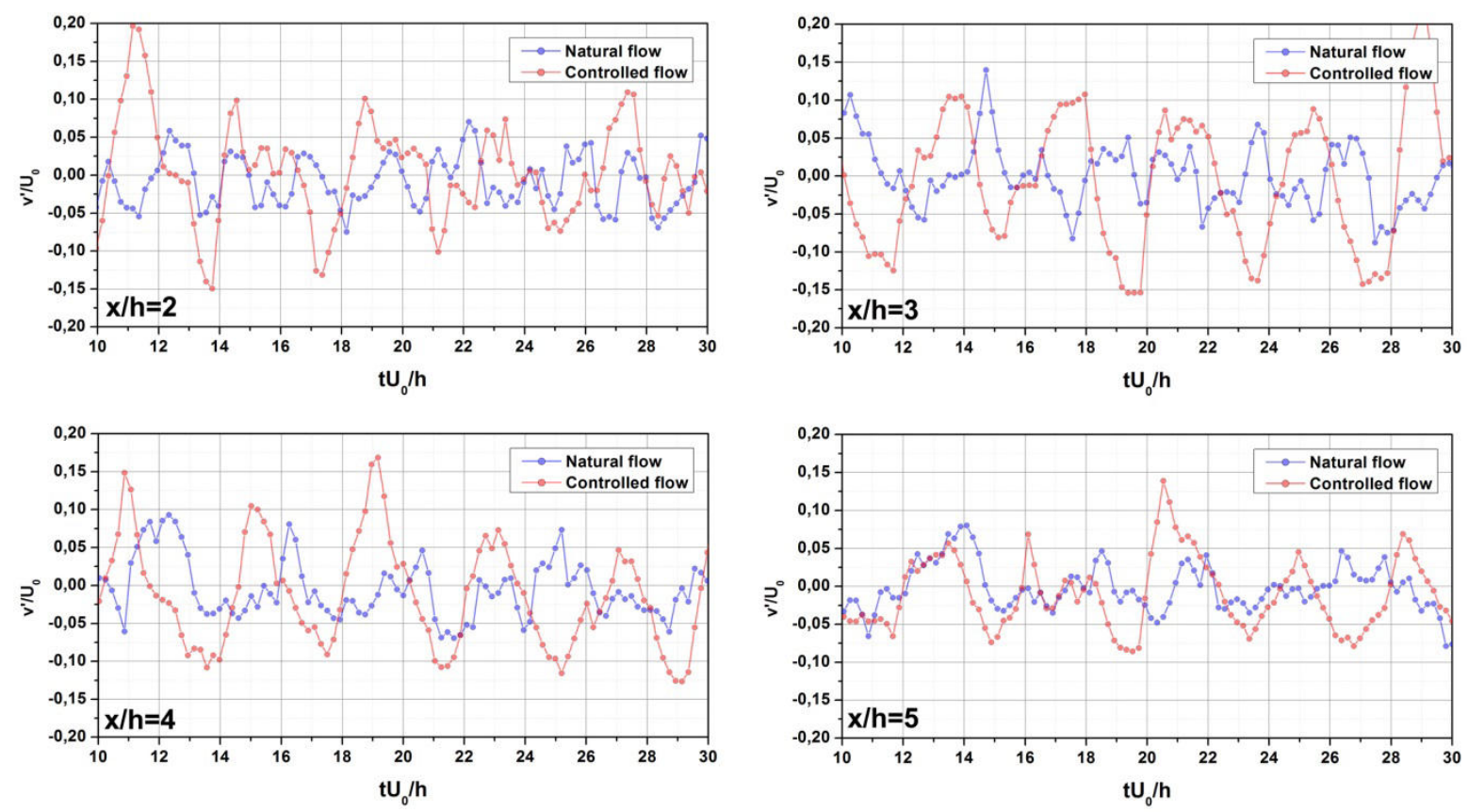

Figure 15 : Time evolution of the fluctuating velocity $v^{\prime}(a t y / h=0)$ at $x / h=2,3,4$ and 5 for the natural flow and periodic forcing at $\mathrm{St}_{\theta}=\mathbf{0 . 0 1 3}$.

An analysis over a short time sequence of a turbulent flow as done in Figure 15 is of interest, but some statistical information related to the vortical flow dynamic can also be determined by inspecting the complete recorded flow sequence. For a more global characterization of the flow dynamic, the power density spectra of the fluctuating component v' at three locations along the shear layer mean centerline are shown in Figure 16. Unfortunately, due to a limited recording time with the PIV, the low frequency fluctuations are not well resolved and subsequently the flapping motion of the separated shear layer cannot be investigated. At the beginning of the shear layer development $(x / h=2)$, the turbulent energy of the baseline flow is small and no discrete frequency dominates. The periodic behavior of the shear layer is not installed at this location. Beyond this position, the turbulent energy is increased, but the frequency content of the turbulent flow is spread over a wide frequency band with maximal amplitude of the PSD observed at low frequency $(7 \mathrm{~Hz}$, presumably the physical frequency related to the flapping of the shear layer). A small amplitude bump can also be observed at about 72 $\mathrm{Hz}$ and its first sub-harmonics, the signature of periodic events at these frequencies. The development of the shear layer under the influence of the plasma discharge gets new insight from the power density spectra analysis. The influence of the actuator is clearly evidenced by the high amplitude peak observed at the forcing frequency $\left(125 \mathrm{~Hz}, \mathrm{St}_{\theta}=0.013\right)$. In the earlier stage of the vortex street formation $(\mathrm{x} / \mathrm{h}<2)$, this frequency signature corresponds to the periodic oscillations of the sheared region. Later, the discrete vortices are formed (see Figure 14), the influence of the forcing frequency is damped while the vortex pairing process becomes significant as indicated by the increase in turbulent energy at the two first sub-harmonics $\left(\mathrm{St}_{\theta} / 2\right.$ and $\mathrm{St}_{\theta} / 4$ at $\mathrm{x} / \mathrm{h}=4$, see Figure 16). The emergence and amplification of coherent flow structures is responsible for the vorticity thickness increases in zone II (Figure 13). At $\mathrm{x} / \mathrm{h}=6$ and beyond this location, the flow is already reattached, but flow structures are still visible past the reattachment location. In the present investigation, the frequency signature at $\mathrm{St}_{\theta}=0.013$ and its two first sub-harmonics emerge from the background turbulence, all of them having a similar amplitude. The presence of several quasi-discrete frequencies after the reattachment point confirms that vortical flow structures persist beyond the reattachment point as discussed in [49]. 


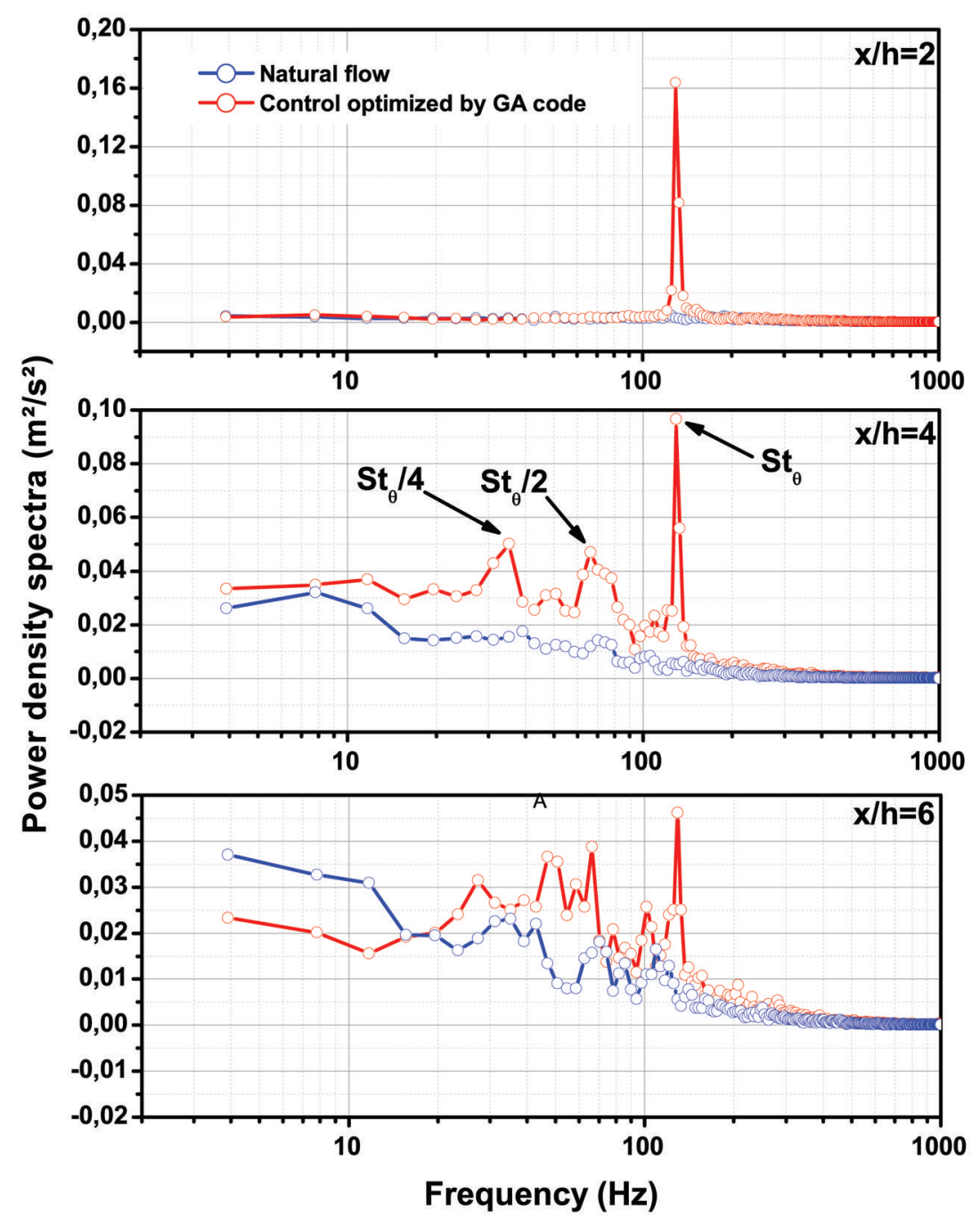

Figure 16: Power density spectra of the fluctuating velocity component $v^{\prime}$ for the natural flow and plasma actuator imposing perturbations at $\mathrm{St}_{\theta}=\mathbf{0 . 0 1 3}$.

The changes in the flow dynamics caused by the surface discharge have been discussed in the previous paragraphs of this paper for mean and time-resolved quantities. In order to add more insight in this study, this final discussion interests in the instantaneous and time-resolved velocity fields for natural and flow control conditions identified by the GA approach. It was observed from Figure 13 that the natural flow presents a linear increase of the vorticity thickness up to a certain position while it is maintained constant further downstream. Moreover, the frequency signature is dominated by low frequency content (the flapping of the separated shear layer [22, 23, 24]) as inferred from Figure 16. An illustration of the evolution of the flow field at different successive instants is given in Figure 17 (natural flow). In this figure, as well as in the following ones, the vortical flow structures embedded in the turbulence of the separated flow are identified by using $\Gamma_{2}$ criterion as it is defined by Graftieaux et al. [50]. The sequence proposed in Figure 17 illustrates partially the complex dynamic observed for the natural flow. It shows that pairing of vortical flow structures occurs at different locations along the shear layer development. A preferential location for the pairing is observed at $\mathrm{x} / \mathrm{h}=3$. At this location, and for the presented time-series, the complete amalgamation of two successive flow structures takes 8 $\mathrm{ms}$ and the resulting structure presents a quite long resident time. The second region of pairing occurs $1 \mathrm{~h}$ downstream of the first one. This region is close to the mean reattachment point and then it is under the influence of the backflow resulting from the impinging of the shear layer on the bottom wall. Vector fields with a finer temporal resolution than Figure 17 are proposed in Figure 18. This sequence 
shows how two vortices (vortices 3 and 4 ) can pair at location $\mathrm{x} / \mathrm{h}=4$. After $1.5 \mathrm{~ms}$, the two structures have merged together to form a discrete coherent rotating region (vortex $3+4$ ). The paring process locally modify the shear rate of parallel streams promoting the formation of a region of mutual interactions between vortical flow structure at $\mathrm{x} / \mathrm{h}=3$ and the novel structure caused by the pairing (see time $\mathrm{t}_{0}+2 \mathrm{~ms}$ in Figure 18). A new vortical flow structure emerges from the mutual interaction between the structures at $\mathrm{x} / \mathrm{h}=3$ and 4 (vortex 5). A part of the turbulent energy contained in the structure located at $\mathrm{x} / \mathrm{h}=3$ is transferred to the newly formed structure. Here, the pairing of the vortical flow structures is accompanied by a splitting mechanism. The cancellation of the growing rate of the vorticity thickness observed at $\mathrm{x} / \mathrm{h}=3.5$ may result from the presence of splitting that contributes to a reduced development of the separated shear layer.

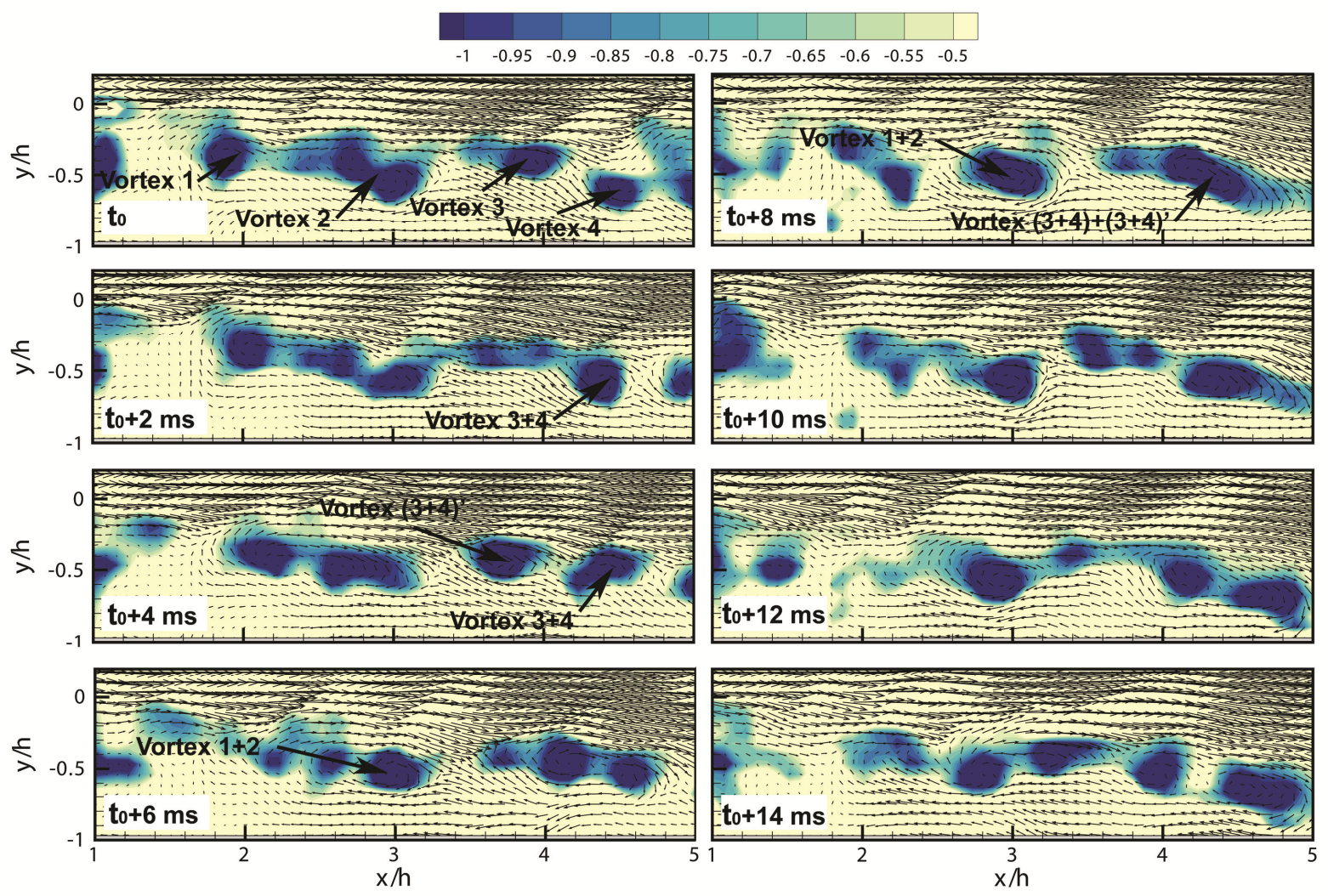

Figure 17 : Illustration of the vortical flow dynamic of the natural flow and pairing process along the separated shear layer. 

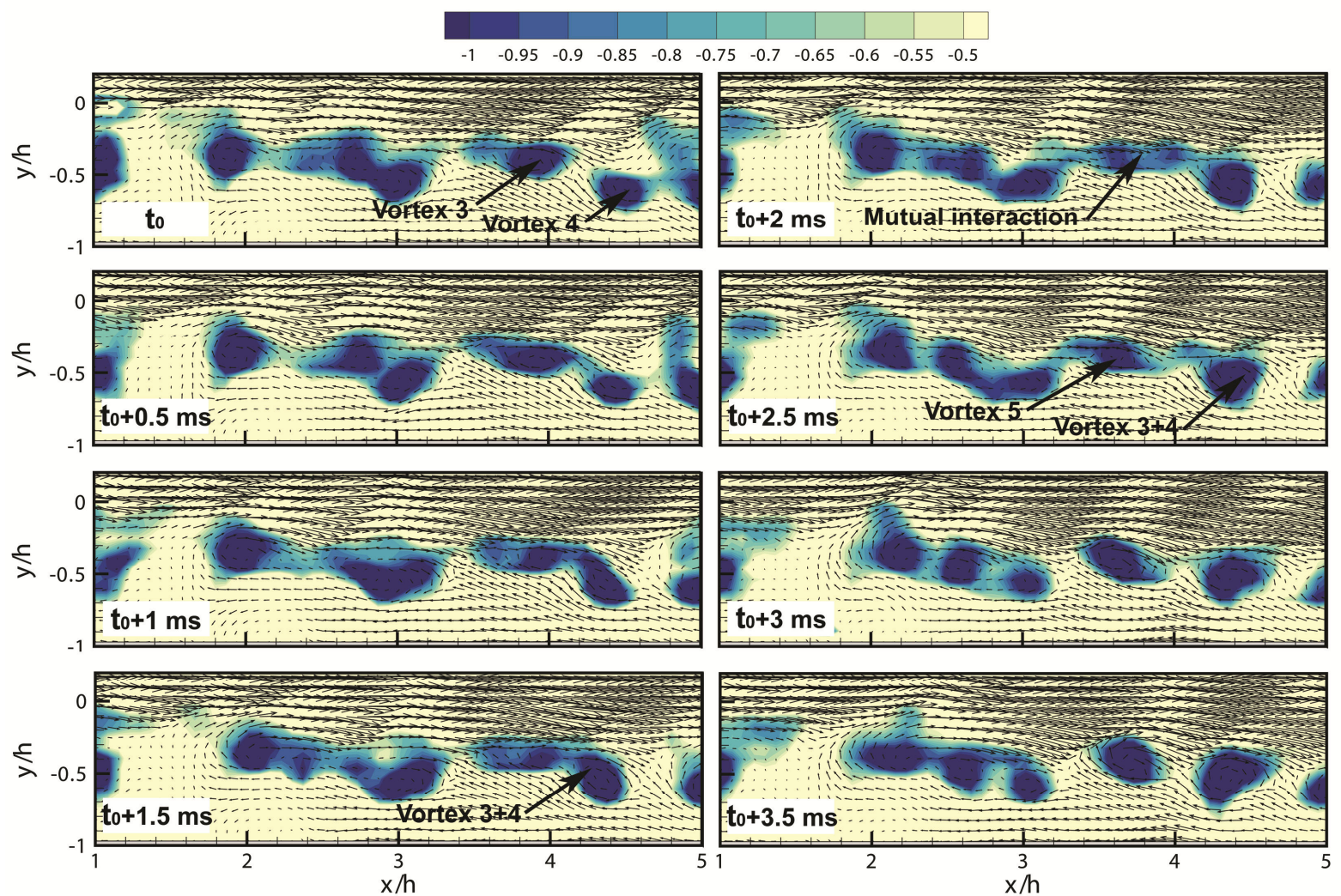

Figure 18 : Illustration of the pairing and splitting of vortical flow structures along the natural separated shear layer.

The time evolution of the flow perturbed by periodic forcing is shown in Figure 19. As already demonstrated all along this paper, the periodic forcing imposed by the plasma discharge reinforces and promotes a higher regularization of the unsteady characteristics of the separated flow. Pairing mechanism is observed and it occurs at a single preferential location (at $\mathrm{x} / \mathrm{h} \approx 4$ ). Two successive vortices (vortices 1 and 2) can merge as it is illustrated by the proposed flow sequence. This pairing results in a single large-scale vortical flow structure (vortex 1+2) that fills the space from the bottom wall up to the upper region of the separated shear layer. The fluid entrains in this large scale coherent structure is high and it results in a transient reattachment point observed just downstream of the structure. The present investigation illustrates how the amalgamation of adjacent vortices can contribute to a larger spreading of the shear layer (as in [20]) and to a shortened recirculating region. Moreover, due to the size of the newly formed flow structure and the presence of a convective flow, the resident time in the recirculating region is reduced and these large-scale coherent flow structures are periodically convected outside the reattached region. The formation by pairing of these large scale structures and their shedding downstream of the reattachment point contribute to the periodic shrinkage of the recirculating region, this being responsible for the large reduction in the mean reattachment location. 

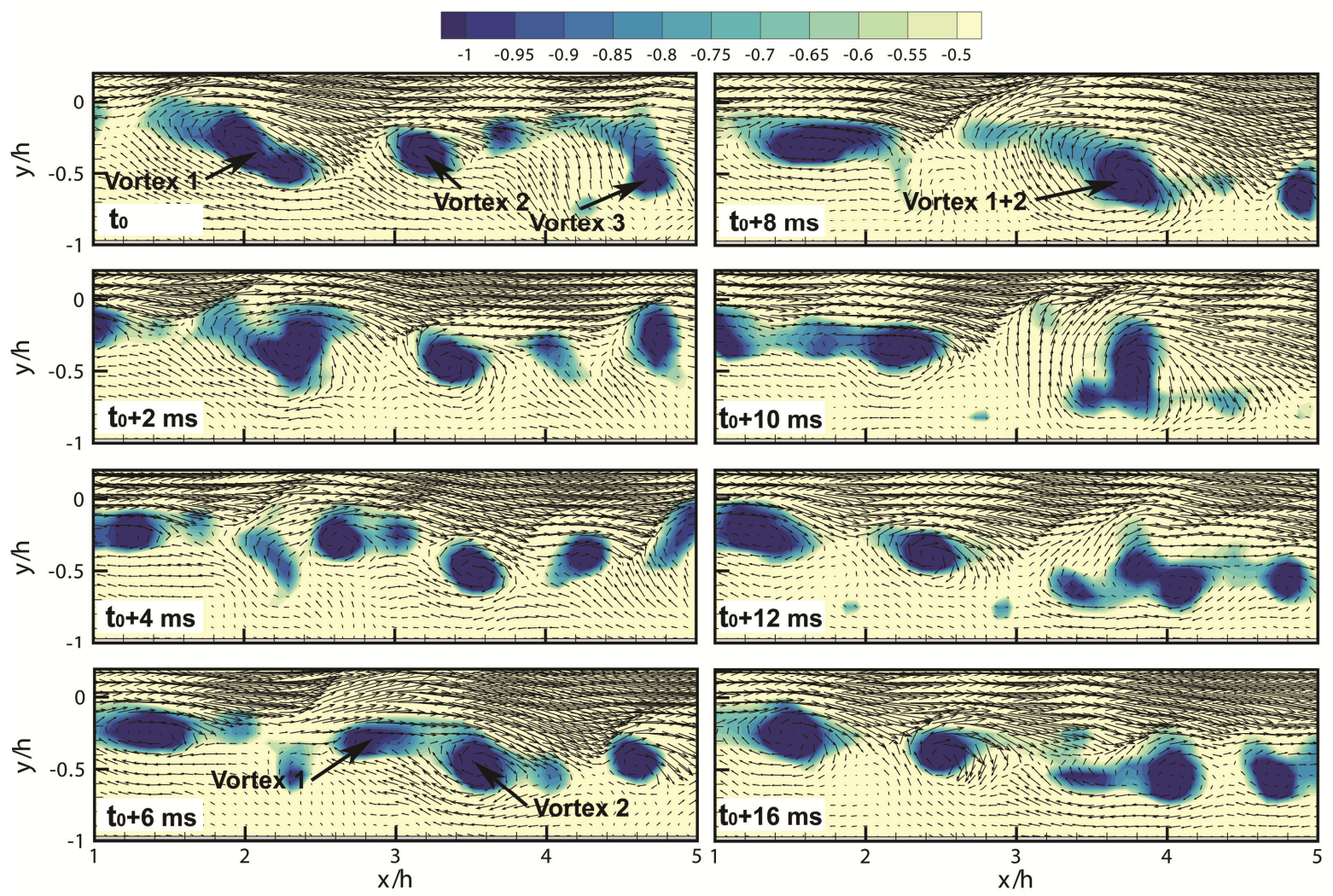

Figure 19 : Illustration of the vortical flow dynamic of the separated shear layer when forced by periodic perturbations produced by a surface plasma discharge.

\section{Concluding remarks}

The present experimental study interests in optimizing the influence of a linear plasma actuator on the vortex development of a separated shear layer formed in the wake of a backward-facing step. Wall pressure and time-resolved measurements are conducted to detect the mean flow characteristics and analyze the influence of the discharge on the flow dynamics. The primary objective was to implement experimentally a genetic algorithm approach for optimizing the control effectiveness.

After a preliminary demonstration of the capability of the GA code for solving inverse problem, this code also success in finding the optimal electrical signal for optimizing the mean reattachment location. The solution identified by the GA code corresponds to periodic perturbations at frequency matching with the natural shear layer mode $\left(\mathrm{St}_{\theta}=0.013\right)$. At such forcing condition, the mean flow recirculation can be reduced by $20 \%$. The periodic forcing caused by the plasma discharge reinforces the flow structures, it regularizes the periodic character of the separated shear flow and it promotes a more energetic pairing mechanism. In particular, time-resolved measurements revealed how the mean flow reattachment is achieved by promoting large-scale coherent flow structures formed by pairing.

In the present investigation, the use of a GA code does not conduce to innovative control scenario. Indeed, the well-known excitation by shear layer mode forcing is the best one identified by the code as one could expect based on a brief survey of the literature. However, the present research confirms for the first time that GA approaches can be easily implemented in experiments. Moreover, optimal control law that considers local flow conditions can also be defined by small modification of the original code. Finally, autonomous seeking of optimal control conditions including multi-input and multi-objective capabilities would be of great interest for the flow control community. Indeed, in 
complex control scenario, for instance when several actuators are considered individually, such algorithms may highlight unseen influence on the flow, reveal new control mechanisms.

\section{Acknowledgment}

This work was supported by the $7^{\text {th }}$ Framework program FP7/2010-2013, MARS (grant agreement $\left.\mathrm{n}^{\circ} 266326\right)$.

\section{References}

1- L.N. Cattafesta, and M. Sheplak 'Actuators for Active Flow Control,' Annual Review of Fluid Mechanics, Vol 43, 2011.

2- E. Moreau, 'Airflow control by non-thermal plasma actuators”, J. Phys.D: Appl. Phys., Vol. 40, 2007

3- T.C. Corke, C.L. Enloe, and S.P. Wilkinson, 'Dielectric Barrier Discharge Plasma Actuators for Flow Control,' Annual Review of Fluid Mechanics, Vol. 42, 2010.

4- Wang, J.-J., Choi, K.-S., Feng, L.-H. and Jukes, T.N., 2013. Recent developments in DBD plasma flow control Progress in Aerospace Sciences. 62, 52-78

5- N. Benard and E. Moreau, 'Electrical and mechanical characteristics of surface AC dielectric barrier discharge plasma actuators applied to airflow control,' Experiments in Fluids, Vol. 55, 2014

6- R. Dawson, and J. Little, 'Characterization of nanosecond pulse driven DBD plasma actuators for aerodynamic flow control' Journal of Applied Physics, Vol. 113, 2013

7- N. Benard, Z. Zouzou, A. Claverie, J. Sotton, and E. Moreau, 'Optical visualization and electrical characterization of fast-rising pulsed DBD for airflow control applications', Journal of Applied Physics, Vol. 111, 2012.

8- T. Michelis, G. Correale, I.B. Popov, M. Kotsonis, D. Ragni, S.J. Hulshoff, and L.M. Veldhuis, 'Disturbance introduced into a laminar boundary layer by a ns-dbd plasma actuator,' AIAA paper 2013-0752

9- Z. Zijie, L. Jiun-Ming, Z. Jianguo, Y.D. Cui, K. Boo Cheong, 'Study of shock and induced flow dynamics by pulsed nanosecond DBD plasma actuators,' AIAA paper 2014-0402.

10- M.L. Post and T.C. Corke, 'Separation Control on High Angle of Attack Airfoil Using Plasma Actuators', AIAA Journal, Vol. 42, 2004.

11- D. Greenblatt, B. Göksel, I. Rechenberg, C. Y. Schüle, D. Romann, and C. O. Paschereit. "Dielectric Barrier Discharge Flow Control at Very Low Flight Reynolds Numbers", AIAA Journal, Vol. 46, 2008.

12- N. Benard, J. Jolibois, E. Moreau 'Lift and drag performances of an axisymmetric airfoil controlled by plasma actuator' Journal of Electrostatics, Vol. 67, 2009

13- J. Little, and S. Samimy, "High-Lift Airfoil Separation Control with Dielectric Barrier Discharge Plasma Actuation", AIAA Journal, Vol. 48, 2010.

14- D.V. Roupassov, A.A. Nikipelov, M.M. Nudnova, and A.Y. Starikovskii, 'Flow separation control by plasma actuator with nanosecond pulsed-periodic discharge', AIAA Journal ,Vol. 47, 2009

15- J. Little, K. Takashima, M. Nishihara, I. Adamovich, and M. Samimy, 'High lift airfoil leading edge separation control with nanosecond pulse driven DBD plasma actuators' AIAA paper 2010-4256

16- I.B. Popov, A. Nikipelov, S. Pancheshnyi, G. Correale, S.J. Hulshoff, L.L.M. Veldhuis, S.Zaidi, and A.Yu. Starikovski, 'Experimental Study and Numerical Simulation of Flow Separation Control with Pulsed Nanosecond Discharge Actuator,' AIAA paper 2013-0572

17- M.A.Z. Hasan, 'The flow over a backward-facing step under controlled perturbation: laminar separation,' Journal of Fluid Mechanics, Vol. 238, 1992.

18- N.J. Cherry, R. Hillier, and P. Latour, 'Unsteady measurements in a separated and reattaching flow', Journal Fluid Mechanics, Vol. 44, 1984.

19- L.M. Hudy, A.M. Naguib, and W.M. Humphreys, 'Wall-pressure-array measurements beneath a separating/reattaching flow region', Physics of Fluids, Vol. 15, 2003.

20- D. Oster, and I. Wygnanski, 'The forced mixing layer between parallel streams,' J. Fluid Mechanics, Vol. 123, pp: 91-130, 1982.

21- C.D. Winant, and F.K. Browand, 'Vortex pairing: the mechanism of turbulent layer growth at moderate Reynolds number,' J. Fluid Mechanics, Vol. 63, pp: 237-255, 1974.

22- D.M. Driver, H.L. Seegmiller and J.G. Marvin, 'Time-dependent behavior of reattaching shear layer', AIAA Journal Vol. 25, 1987

23- I. Lee and H.J. Sung, 'Characteristics of wall pressure fluctuations in separated and reattaching zone of a turbulent separation bubble', Experiments in Fluids, Vol. 30, 2001

24- M. Kya, and K. Sasaki, 'Structure of large-scale vortices and unsteady reverse flow in the reattaching zone of a turbulent separation bubble', Journal of Fluid Mechanics, Vol. 154, 1985.

25- J. d'Adamo, R. Sosa, and G. Artana, , 'Active Control of a Backward Facing Step Flow With Plasma Actuators,' Journal of Fluid Engineering, Vol. 136, 2014

26- N. Benard, P. Sujar-Garrido, K.D. Bayoda, J.P. Bonnet, and E. Moreau, 'Pulsed dielectric barrier discharge for manipulation of turbulent flow downstream a backward-facing-step,' AIAA paper 2014-1127

27- P. Sujar-Garrido, N. Benard, J.P. Bonnet, and E. Moreau, 'Dielectric barrier discharge plasma actuator to control turbulent flow downstream of a backward-facing step,' Experiments in Fluids, Vol. 56, 2015. 
28- H. Zare-Behtash, K. Kontis, and S. roy, 'Flow control at subsonic speeds using serpentine plasma actuators, AIAA paper 2014-2812

29- L. Huang, G. Huang, R. LeBeau and T. Hauser, 'Optimization of airfoil flow control using a genetic algorithm with diversity control,' J. Aircraft, Vol. 44, 2007

30- A. Hilgers, and B.J. Boersma, 'Optimization of turbulent jet mixing,' Fluid Dynamics Research, Vol. 29, 2001

31- T.K. Sengupta, K. Deb, and S.B. Tala, 'Control of flow using genetic algorithm for a circular cylinder executing rotary oscillation,' Computers and Fluids, Vol. 36, 2007

32- T. Watanabe, T. Tatsukawa, A.L. Jaimes, H. Aono, T. Nonomura, A. Oyama, and K. Fujii, 'Many-objective evolutionary computation for optimization of separated-flow control using a DBD plasma actuator,' Evolutionary Computation (CEC) IEEE, 2849 - 2854, 2014

33- N Gautier, T Duriez, JL Aider, B Noack, M Segond, M Abel, 'Closed-loop separation control using machine learning,' Journal of Fluid Mechanics, Vol. 770, 2015

34- T. Duriez, V. Parezanovic, J.C. Laurentie, C. Fourment, J. Delville, J.P. Bonnet, L. Cordier, B.R. Noack, et al. 'Closed-loop control of experimental shear flows using machine learning,' AIAA paper 2014

35- N.N. Mansour, F. Hussain, and J. Buell, 'Subharmonic resonance in a plane mixing layer', 1988 Summer Workshop Report of CTR, Stanford U., pp. 3-18, 1988.

36- U.K. Kaul, 'First principles based PID control of mixing layer: Role of inflow perturbation spectrum', AIAA paper 2014-2222

37- S. Chun, Y.Z. Liu and H.J. Sung, 'Wall pressure fluctuations of a turbulent separated and reattaching flow affected by an unsteady wake,' Experiments in Fluids, Vol. 37, 2004

38- S. Bandyopadhyay and S. Saha, 'Unsupervised classification,' Springer-Verlag, 2013.

39- D.S. Lee, L.F. Gonzalez, J. Periaux, and G. Bugeda, Double Shock Control Bump Design Optimisation Using Hybridised Evolutionary Algorithms. Special Issue Journal of Aerospace Engineering, Vol. 225, 2011.

40- D.S. Lee, C. Morillo, G. Bugeda, S. Oller, and E. Onate, Multilayered Composite Structure Design Optimisation using Distributed/Parallel Multi-Objective Evolutionary Algorithms, Journal of Composite Structures, 2011.

41- D.S. Lee, J. Periaux, E. Onate, L.F. Gonzalez, Advanced Computational Intelligence System for Inverse Aeronautical Design Optimisation, International Conference on Advanced Software Engineering (ICASE-11), Proceedings of the 9th IEEE International Symposium on Parallel and Distributed Processing with Applications Workshops, ISPAW, 2011.

42- D. S. Lee, J. Periaux, and L. F. Gonzalez, UAS Mission Path Planning System (MPPS) Using Hybrid-Game Coupled To Multi-Objective Optimiser. Journal of Dynamic Systems, Measurement and Control, DS-09-1135.

43- K.B. Chun, and H.J. Sung, 'Control of turbulent separated flow over a backward-facing step by local forcing,' Experiments in Fluids, Vol. 21, pp: 417-426, 1996.

44- C.M. Ho, and L.S. Huang, 'Subharmonics and vortex merging in mixing layers', Journal of Fluid Mechanics, Vol. 119,1982

45- I. Wygnanski, and I. Weisbrot, 'On the pairing process in an excited plane turbulent mixing layer,' in Turbulence Management and Relaminarisation ed. Liepmann and Narasimha, Springer book, 1988

46- U.K. Kaul, 'First principle based PID control of mixing layer: Role of inflow perturbation spectrum,' AIAA paper 2014-2222.

47- H.Q. Zhang, and W. Shu, 'Numerical simulations of vortex merging and vortex splitting in mixing layer,' Science in China, Vol. 33, 1990

48- G.L. Brown, and A. Roshko, 'On density effects and large structure in turbulent mixing layers,' Journal of Fluid Mechanics, Vol. 64, 1974.

49- T.R. Troutt, B. Scheelke and T.R. Norman,'Organized structures in a reattaching separated flow field,' J. Fluid Mechanics, Vol. 143, pp: 413-427, 1984

50- L. Graftieaux, M. Michard, and N. Grosjean, 'Combining PIV, POD and vortex identification algorithms for the study of unsteady turbulent swirling flows', Measurements Sciences and Technology, Vol. 12, 1422, 2001 